\title{
VII. Die achtziger Jahre: Konfrontation und Kulmination
}

\section{Die frühen achtziger Jahre: Günzburg und der Fall Mengele im Spannungsfeld deutschen Geschichtsbewußtseins}

\section{Die 1970er Jabre und die TV-Serie "Holocaust“}

Nach den großen Prozessen in der ersten Hälfte der 1960er Jahre, der lautstarken Kritik an der bis dahin in der Bundesrepublik geübten Praxis der Vergangenheitsbewältigung (oder vielmehr Nicht-Bewältigung) durch die 68er-Bewegung und der dritten Verjährungsdebatte im Bundestag 1969 wurde es wieder etwas ruhiger um die deutsche Vergangenheit. ${ }^{1}$ Aleida Assmann teilt die Geschichte der deutschen Vergangenheitsbewältigung in drei Phasen, deren zweite sie von 1958 bis 1984 als Phase der „Kritik der Vergangenheitsbewältigung“2 definiert. Daß dies über beinahe drei Jahrzehnte hinweg, die der Bundesrepublik einschneidende soziale Veränderungen brachten, kein kontinuierlicher, in einheitlichen Bahnen verlaufender Prozeß sein konnte, ist evident. Vielmehr vollzog sich die "deutsche Erinnerungsgeschichte [...] mit Vorliebe in Sprüngen und Eruptionen“, und „seit der Mitte der achtziger Jahre“, so Assmann, war der „bevorzugte Anstoß zu solcher Erinnerung der Skandal"3. Das ist insofern richtig, als sich zu diesem Zeitpunkt eine ganze Reihe solcher Skandale - nach Assmann die "Erregung öffentlichen Ärgernisses“ oder „öffentlicher Aufmerksamkeit" - ereigneten ${ }^{4}$, doch war dies nicht allein ein Phänomen der 1980er-Jahre: diese neuerliche „Eruption“ der deutschen Vergangenheitsbewältigung war nicht die erste und sollte nicht die letzte bleiben. Beginnen müßte eine Aufzählung in der unmittelbaren Nachkriegszeit und den frühen Jahren der Bundesrepublik ${ }^{5}$ und - sicherlich nur vorläufig - enden mit der GoldhagenDebatte, der Kontroverse um die Wehrmachtsausstellung und dem Walser/BubisStreit. Auch die großen Prozesse der sechziger Jahre, vor allem der Eichmann- und der Auschwitz-Prozeß, waren solche „Skandale“. Sie hatten die „allgemeine Kenntnis über den Genozid erweitert und das Bewußtsein einer größeren Öffentlichkeit für das Ausmaß der verübten Verbrechen zumindest ansatzweise sensibilisiert"6.

1 Vgl. Assmann/Frevert, Geschichtsvergessenheit, S. 144. Vgl. zu den Verjährungsdebatten des Bundestages: Reichel, Vergangenheitsbewältigung, S. 185-198.

2 Assmann/Frevert, Geschichtsvergessenheit, S. 144.

3 Dieses und die folgenden Zitate: Ebenda, S. 21.

4 Assmann nennt hier Reagans Bitburg-Besuch (1985), den Historiker-Streit (1986) und die Jenninger-Rede (1987). Auch den „Skandal“ der Auffindung der Leiche Mengeles könnte man in diese Aufzählung einreihen.

5 Vgl. dazu Frei, Vergangenheitspolitik.

6 Reichel, Vergangenheitsbewältigung, S. 205. 
Die 1970er Jahre blieben lange Zeit ohne solch große „Skandale“, die „Sprünge und Eruptionen" blieben aus; die erhöhte Sensibilisierung aber blieb erhalten. Auch um Josef Mengele und Günzburg wurde es bald wieder ruhiger. Nach dem eruptiven Schub des Auschwitz-Prozesses, der die öffentliche Aufmerksamkeit im Fall Mengele erregt und auf die Stadt gelenkt hatte, ebbte das Interesse der Öffentlichkeit an der Heimatstadt des gesuchten KZ-Arztes schnell wieder ab, ohne daß ihre angebliche dunkle Rolle als verschworene Gemeinschaft, die den Massenmörder schützte, in Vergessenheit geriet. Dafür sorgten auch die Nazi-Jäger, allen voran Simon Wiesenthal, der in Interviews selten vergaß, darauf hinzuweisen, daß Mengele ein „schwerreicher Mann [...] mit vielen Freunden und einer großen, intakten Familie" sei, die ihn mit allem „im Überfluß" versorge; das Geld stamme natürlich aus Günzburg, einem Ort, in dem alle „direkt oder indirekt von der Mengele-Firma abhängig "7 seien. Nationale und internationale Zeitungen berichteten weiterhin über die neuesten Gerüchte im Fall Mengele. Kontinuierlich wurden alte Geschichten variiert und an neuen Legenden gestrickt, immer wieder war von spektakulären Mengele-Sichtungen die Rede. Der Mythos wuchs weiter, der Arzt von Auschwitz aber blieb verschwunden. ${ }^{8}$

Dies alles berührte Günzburg in den 1970er Jahren wenig. Erst zu Beginn der 1980er Jahre sollte das Thema Josef Mengele wieder auf der lokalen Tagesordnung erscheinen. Mittlerweile hatte ein neuer "Skandal" die deutsche Erinnerungslandschaft erschüttert: Im Januar 1979 war der vierteilige Fernsehfilm „Holocaust“ ausgestrahlt worden, der überaus publikumswirksam und melodramatisch die individuellen Schicksale der jüdischen Familie Weiß und des SS-Offiziers Dorf erzählte. Der Film erschütterte Millionen, Reichweite und Wirkung waren enorm. ${ }^{9}$ Schon

7 Das Netz um SS-Arzt Mengele wird immer enger!, in: Bild am Sonntag, 22. 6. 1980. Dieses Interview ist geradezu beispielhaft für die Meldungen, die Wiesenthal in den 1970er und frühen 1980er Jahren in der Presse lancierte.

Wiesenthal konnte dem Journalisten in diesem Gespräch außerdem von angeblichen „Familientreffen [...] in Mailand und auf den Bahamas" berichten. „Obwohl ich gegenwärtig seine Spur verloren habe", zeigte er sich erstaunlich gut informiert über den physischen und psychischen Zustand Mengeles und teilte mit, „daß er Kreislaufbeschwerden hat, nie ohne vier ausgesuchte Leibwächter reist und immer häufiger nach schrecklichen Wutanfällen körperliche Zusammenbrüche erleidet." Sogar den Grund für Marthas Rückkehr nach Europa wollte Wiesenthal kennen: „Sie ertrug Mengeles Unruhe nicht mehr, die ihn niemals länger als drei Wochen im selben Bett schlafen läßt". Auch Mengeles momentane Freizeitbeschäftigung wollte Wiesenthal kennen: „Sie dürfen mich nicht fragen, woher ich es weiß, aber - ich weiß es: Josef Mengele bastelt seit einigen Monaten an seiner Verteidigungsrede." Ähnliche Beispiele finden sich in: Posner/Ware, Mengele, S. 354.

8 Vgl. z.B.: „Endlich: Wir haben Mengele entdeckt“, in: Bild am Sonntag, 14. 2. 1971 und Bormans Gang: World exclusive challenge! To Nazi-hunter Simon Wiesenthal... Now follow my steps to the Doctor of Death und Bormanns Gang. I find the Nazi Doctor of Death, in: Daily Express, 1. 12. 1972.

9 Die Einschaltquoten stiegen über den Sendezeitraum der vier Abende von $31 \%$ auf $40 \%$, und $48 \%$ der Gesamtbevölkerung im Alter über 14 Jahre hatten mindestens einen Teil der Serie gesehen; Kommentatoren verglichen dic Wirkung des Films auf die öffentliche Diskussion mit der des Tagebuchs der Anne Frank. Vgl. Marcuse, Legacies, S. 343.

Erst seit diesem Zeitpunkt wird die Ermordung der europäischen Juden häufig unter dem nicht unproblematischen Begriff Holocaust zusammengefaßt. Er ist vor $1979 \mathrm{im}$ Duden oder in deutschen Fremdwörterbüchern nicht nachweisbar. Er ist griechisch-lateinischen Ursprungs (holocausta, von griech. "holos" = ganz und lat. „caustos" = gebrannt). Der Termi- 
die vielbeachtete Ausstrahlung in den USA, in England, Belgien und Israel im Jahr zuvor hatte dazu geführt, daß der Jahrestag der „Reichskristallnacht“, die sich 1978 zum vierzigsten Mal jährte, in den Zeitungen thematisiert und die Verfolgung der Juden in Deutschland so intensiv diskutiert wurde wie niemals zuvor. ${ }^{10}$ Dabei war der Film kein grundlegender Wendepunkt in der Auseinandersetzung mit der eigenen Vergangenheit, sondern vielmehr der erste Höhepunkt einer Entwicklung, die, getragen von einer neuen Generation junger Deutscher, schon in den siebziger Jahren begonnen hatte. ${ }^{11}$ Animiert durch die in „Holocaust" geschilderten Individualschicksale und weniger von Tabus und eigener Betroffenheit belastet als Eltern und Großeltern, interessierte sich diese Generation besonders für die Alltagsgeschichte des Nationalsozialismus, auch und gerade im lokalhistorischen Kontext. ${ }^{12}$ Dies führte zu einer Veränderung des gesamtgesellschaftlichen Klimas im Umgang mit der Vergangenheit, die schließlich in die "Geschichtsversessenheit" ${ }^{13}$ der 1980er und 1990er Jahre mündete.

\section{Anstoß von außen: Hanne Hiob und die "Idylle einer deutschen Kleinstadt"}

Obwohl der Fall Mengele 1979 international erneut Schlagzeilen gemacht hatte ${ }^{14}$, dauerte es bis Ende 1982, bis Günzburg wieder mit ihm konfrontiert wurde. Die deutsche Linke hatte "Holocaust" aus mehreren Gründen kritisiert. Einer davon war der Vorwurf, der Film impliziere, daß der Nationalsozialismus 1945 geendet

nus rückt die Massenvernichtung der europäischen Juden (andere Opfergruppen bleiben meist ausgeklammert) in einen quasi-religiösen Zusammenhang mit alttestamentarischen Opferriten: Der Begriff Holocautatio/in meint im Griechischen die Darbringung eines Brandopfers, in der Regel in Form eines Tieres, das auf einem Altar verbrannt wird, und hat spätestens im 12. Jahrhundert auch Eingang ins Kirchenlatein (holocaustum) gefunden. In Israel wird statt "Holocaust“ in der Regel der hebräische Begriff „Shoa" verwendet, was so viel wie Katastrophe oder Verwüstung bedeutet. Vgl. Yonan, Gabriele, Jehovas Zeugen. Opfer unter zwei deutschen Diktaturen. 1933-1945. 1949-1989, Berlin 1999, S. 39-41.

10 Vgl. Marcuse, Legacies, S. $344 \mathrm{f}$.

11 Vgl. Reichel, Vergangenheitsbewältigung, S. 205, und Marcuse, Legacies, S. 345.

12 Verwiesen sei hier nur auf die Geschichtswerkstätten, die sich in vielen Städten mit der lokalen NS-Geschichte auseinandersetzten, und den Schülerwettbewerb Deutsche Geschichte um den Preis des Bundespräsidenten, dessen Themenstellungen deutlich das veränderte Interesse zeigen: dominierten in den 1970er Jahren noch Themen wie „Die Revolution von 1848“ (1974), „Die Arbeitswelt" (1977) oder „Freizeit" (1979), so hieß das Thema von 1980 bis 1985 „Alltag im Nationalsozialismus“, unterteilt in die Perioden Vorkriegs-, Kriegs- und Nachkriegszeit. Die Teilnehmerzahl, die 19793995 betragen hatte, verdreifachte sich 1980 auf 12843 (um dann allerdings wieder zu sinken). Die wohl bekanntesten Teilnehmer des Wettbewerbs sind Anna Rosmus (1980/81), die sich mit der NS-Geschichte ihrer Heimatstadt Passau beschäftigte und deswegen Morddrohungen erhielt sowie Michael Brenner, der über das jüdische Leben in Weiden forschte und 1997 als einer der jüngsten deutschen Professoren auf den neuen Lehrstuhl für Jüdische Geschichte und Kultur der Universität München berufen wurde. Vgl. auch Marcuse, Legacies, S. 349-351.

13 Assmann/Frevert, Geschichtsvergessenheit, S. 10.

14 Nach einer Fernsehsendung der CBS, in der erstmals Belege dafür vorgelegt wurden, daß Mengele sich zumindest zeitweise in Paraguay aufgehalten hatte, sah sich die paraguayanische Regierung unter dem Druck der USA erstmals genötigt, zu erklären, daß Mengele sich „seit 1960 nicht mehr im Lande aufgehalten“ habe; gleichzeitig wurde ihm mit dieser Begründung die Staatsangehörigkeit entzogen - zu diesem Zeitpunkt war er allerdings bereits tot. Vgl. Posner/Ware, Mengele, S. 356-358. 
habe; er versäume es, auf immer noch vorhandene faschistische Strukturen und Tendenzen im kapitalistischen Deutschland hinzuweisen. ${ }^{15}$ Im Oktober 1982 druckte die in München erscheinende maoistische Kommunistische Arbeiterzeitung einen Artikel mit dem Titel „Idylle einer deutschen Kleinstadt“, der aus der Feder Hanne Hiobs ${ }^{16}$, der Tochter Bertolt Brechts, stammte und Günzburg in diesem Sinne als Beleg und negatives Beispiel instrumentalisierte.

Der Artikel teilte Günzburg entlang starrer, klassenkämpferischer Interpretationslinien in zwei Lager: Die proletarische Unterstadt und die Oberstadt der „Notare, Apotheker, Beamten, Ärzte“, der „Küchles, die keine Messe auslassen [...], denn sie backen in ihrer Oblatenfabrik Hostien“ und der „Fabrikantenfamilie Mengele" 17 . Dort, in der Oberstadt, sei 1946 bis 1950 Josef Mengele ein- und ausgegangen, „die Günzburger freundlich grüßend auf der Straße“, schrieb Hanne Hiob, fast so, als sei sie dabeigewesen. Natürlich habe ihn niemand angezeigt, denn die „ganze, verbissen schweigende Oberstadt“ sei „versippt, verschwägert und allesamt abhängig von der Familie Mengele“.

„Der Bevölkerung Günzburgs eine gemeinsame Haltung pro Mengele zu unterstellen" 18 fand auch Hanne Hiob absurd, denn: „Die Haltung der Oberstadt ist nicht die Haltung der Unterstadt“, die natürlich nicht „freiwillig arbeitet [...] für die Mengeles und das flotte Leben des Massenmörders Josef Mengele in Südamerika." Leider war sich diese Unterstadt des ausgeklügelten Systems ihrer Ausbeutung keineswegs bewuß $\mathrm{t}^{19}$ : Sie wollte „dem ihr unbekannten Neugierigen“, der doch nur ihr

15 Vgl. Marcuse, Legacies, S. 345.

16 Geb. 12. 3. 1923 in München; Mutter: Marianne Zoff; 1942 -1944 als Tänzerin in Salzburg, 1943/44 2 Filmrollen; bis 1976 Theaterschauspielerin, u.a. in Berlin, Hamburg, Zürich und München; seit 1976 besonders ausgeprägtes politisches Engagement, insb. im Zusammenhang mit dem Werk ihres Vaters Berthold Brecht; in den 1980er Jahren mehrere Filme im Fernsehen der DDR. 1979/80 hatte Hiob durch den antifaschistischen "anachronistischen Zug" gegen die Wahl von Karl Carstens zum Bundespräsidenten und die Kanzlerkandidatur von Franz Josef Strauß Aufsehen erregt.

17 Dieses und die folgenden Zitate: Idylle einer deutschen Kleinstadt, in: KAZ, 23. 10. 1982.

18 Hiob zitiert dies ohne Quellenangabe aus: In Günzburg blieb die Zeit nicht stehen, in: GZ, 16. 7. 1964.

19 Hanne Hiob tut sich in ihrem Artikel einigermaßen schwer, die Firma Mengele in das starre Schema des Klassenkampfes zu pressen. So muß sie feststellen, es habe „von 1961 bis 1979 nicht mal einen einzigen Arbeitsgerichtsprozeß“ gegeben, obwohl doch „80\% der Arbeiter [...] beim Mengele [...] gewerkschaftlich organisiert" sind. Grund dafür ist offenbar ein wenig klassenbewußter Betriebsratsvorsitzender von der SPD, der nur zu sagen weiß, die Arbeiter seien „voll integriert in den Betrieb, voll!“. Doch, weil nicht sein kann, was nicht sein darf, gibt es den Klassenantagonismus natürlich auch in der Firma Mengele. Dies versucht sie an Hand eines Beispiels zu belegen, mit dem der Sozialdemokrat wohl das gute Betriebsklima darzustellen versuchte: „Wenn ein Arbeiter Geburtstag hatte, kaufte er ein Tragl Bier. Dann kam [...] auch der Alois Mengele, setzte sich auf die Kiste und [...] unterhielt sich dabei mit ihm wie mit seinesgleichen." Doch der Kapitalist kam nicht, um dem Arbeiter zu gratulieren. Er kam, so Hiobs Wertung, um ,eins von dessen Bieren“ zu trinken.

Die Unterstädter hatten offenbar nicht begriffen, daß etwa der „Ruth-Mengele-Kindergarten“ von dem Kapitalisten nur deshalb gebaut worden war, um „den Kindern der Unterstadt [...] ihre Zukunft abzugewöhnen“. "Aufgemuckt" hatten sie nur einmal, 1970, als sie „den Dr. Köppler, einen SPD-Mann“, ins Rathaus wählten - nur zwei Jahre später habe der „dann den Alois Mengele zum Ehrenbürger gemacht" und die Günzburger Arbeiter damit geradezu verraten: Wegen dieser „vaterländischen Gesinnung“ hätten „sie ihn allerdings nicht 
Bestes will, „nicht erzählen“. Also suchte Hiob in der Geschichte und stieß dort auf den von den Nationalsozialisten in den Selbstmord getriebenen Otto Geiselhart, zwar von der SPD, doch immerhin ein Märtyrer. Um mutige Leute wie ihn loszuwerden, hätten sich die Kapitalisten den Nationalsozialismus geschaffen.

Von nun an folgte die Argumentation dem sozialistischen Geschichtsbild des historischen Materialismus. Darin eingebettet offenbarte Hanne Hiob teils gut recherchierte Kenntnisse zu den Verhältnissen in Günzburg und der Biographie Josef Mengeles, die jedoch oft durch überzogene Polemik und allzu starre Einzwängung in ein vorgefertigtes ideologisches Korsett entwertet wurden - und natürlich fanden sich auch hier die unvermeidlichen Versatzstücke des Mengele-Mythos. Ihr Fazit: Die Oberstadt sei „die geistige und sittliche Welt des Josef Mengele“, ihn „schützt [sie] bis heute und schickt ihm ihre Schecks“. Um gegen die "widerspenstige Unterstadt weiter existieren zu können", habe die Oberstadt - heute wie damals - die "[Josef] Mengeles und die anderen, stets zu allem bereiten Richter, Generäle, Vollzugsbeamten, Redakteure, Pfaffen, Gelehrten, Ärzte und Henker“ gebraucht. „Daß die idyllische Provinzstadt so ungerührt bis heute den Juden, den Arbeiter, den Oppositionellen hassen und den Judenwürger ehren und nähren kann", daß die Oberstadt überhaupt „den Massenmörder Mengele hervorgebracht hat, daran ist die Existenz des Hauses Siemens und der anderen schuld“, schließlich hätten „die Interessen der Firmen Siemens, IG Farben, Krupp und Thyssen direkt zur Rampe von Auschwitz" geführt. Da war es nur folgerichtig, daß der Sohn des Kapitalisten Mengele, der die Oberstadt beherrschte, einer der „schlimmsten Mörder des KZStaates" war. So hatten die Kapitalisten und Faschisten der Oberstadt Josef Mengele hervorgebracht, und ihr Produkt war der beste Beweis, daß sie Kapitalisten und Faschisten waren.

Und diese Kapitalisten und Faschisten herrschten noch immer in der Oberstadt. So wurde Günzburg zum negativen Vorzeigeobjekt für ein kapitalistisches System, in dem die Arbeiterklasse weiterhin ausgebeutet wurde und dessen Gerichte „Oberstadt-Gerichte“ waren, die "Oberstadt-Recht" sprachen. Die Oberstadt war überall, und „angeklagt mit Mengele ist ein Staat ${ }^{\text {" } 20}$ und seine faschistischen Tendenzen, verborgen hinter der "Idylle einer deutschen Kleinstadt", die „allen idyllischen Kleinstädten in unserem Land gleichzustellen“ 21 ist. „Die Idylle von Günzburg ist der Faschismus [...] von heute. "22 Leider waren die Arbeiter nicht aufgeklärt genug, um zu erkennen, daß sie von den faschistischen Kapitalisten der Oberstädte ausgebeutet wurden. Deshalb bedurfte es der Provokation, und deshalb kam Hanne Hiob nach Günzburg, um die Menschen dort über Josef Mengele aufzuklären und ihnen damit die Augen zu öffnen über die Oberstadt, um dafür zu sorgen, daß „auch hier [...] die Unterstadt aufstehen, Anklage erheben und ihr Recht suchen“23 wird.

gewählt“. Dieser Passus crinnert stark an die Sozialfaschismusvorwürfe, die die KPD in der Weimarer Republik gegen die Sozialdemokraten erhob.

20 Hiob, Idylle, S. 26. In dieser Broschüre faßte Hanne Hiob die wichtigsten Aussagen ihres $\mathrm{KAZ}$-Artikels nochmals zusammen, ergänzt um eine Schilderung des Informationsstandes in Günzburg.

21 Hiob, Idylle, S. 20.

22 Ebenda.

23 Idylle einer deutschen Kleinstadt, in: KAZ, 23. 10. 1982. 
„So ganz wohl war's der Stadtverwaltung nicht bei der Entscheidung über den Antrag “24 Hanne Hiobs und ihrer Begleiterin Brigitte Zuber, „einen Informationsstand zum Thema Josef Mengele" zu errichten. Grundsätzliche Zweifel an der Genehmigung habe es nicht gegeben, so die GZ, aber im Ordnungsamt befürchtete man offenbar, es könne zu einer „erregten Menschenansammlung" kommen. Die amtlichen Befürchtungen zeigen, daß Hiob und Zuber ein Thema aufgriffen, das man durchaus für brisant hielt. Tatsächlich herrschte am 23. November 1982, einem Dienstag, während der Mittagsstunden „ein regelrechter Großandrang“ an dem Stand der beiden Kommunistinnen, und es wurde „eifrig diskutiert".

Es seien "alle Meinungen vertreten“ gewesen, so das Fazit Brigitte Zubers nach drei Stunden. Auf die „Provokation“, die „bewußt einkalkuliert“ war, wie einer der Helfer der beiden Kommunistinnen bestätigte, folgten natürlich Gegenprovokationen. „Ganz nah, ganz freundlich“ habe ihr ein Mann „ins Gesicht“ gesagt: „Mengele ist der schönste Name auf der ganzen Welt!" 25 , schrieb Hanne Hiob zwei Jahre später, und: „Dachau gehört wieder eingeheizt, und dann alle rein mit euch. Ihr gehört weg!“26 Doch solche Ausfälligkeiten „stießen in den Reihen der um den Stand stehenden Bürger selbst auf empörten Widerspruch“27. Es entwickelte sich eine „Bürgerdiskussion“, wie sie „, in den 70er Jahren [... ] in Günzburg noch nicht möglich gewesen“ wäre, war sich Brigitte Zuber sicher. „Das ist inzwischen offener geworden."

Damit dürfte sie Recht gehabt haben. Das steigende Interesse von Teilen der deutschen Bevölkerung an den Verbrechen des Nationalsozialismus war auch an Günzburg nicht vorübergegangen. „Viel Zuspruch“ hätten die Initiatoren für ihren Versuch erhalten, „die Günzburger über Werdegang und Schuld des [...] Josef Mengele zu informieren“. Walter Roller, der das Geschehen einige Tage später im Lokalteil der GZ ausführlich kommentierte, konstatierte denn auch ein „gerütteltes Maß an Informationsdefizit, das es im Sinne der dringend notwendigen Aufbereitung dieses historischen Zeitabschnittes" 28 auszugleichen gelte.

So mancher Günzburger hatte dem Informationsstand aber auch ablehnend gegenübergestanden. "Was mit den Deutschen passiert ist, darüber redet keiner"29, lautete einer der Einwände, oder „Mengele hat doch nur wie jeder andere Krieger seine Pflicht erfüllt." Auch, daß "nach 40 Jahren die Dinge endlich ruhen" sollten, war zu hören. Dem widersprach Roller, man dürfe und müsse sich „gerade auch in Günzburg über Josef Mengele unterhalten “30; dies sei Teil „jener Vergangenheitsbewältigung, die [...] nicht befriedigend geleistet wird“, denn „hier gibt es, so schmerzlich dies für manchen sein mag, noch etliches zu tun“. "Schweigen oder gar aggressive Abwehrhaltungen sind hier fehl am Platz", hielt er all denen entgegen, „die der Konfrontation [...] ausweichen und jede Aktion dieser Art für verachtens-

24 Dieses und die folgenden Zitate: „Das geht Günzburg am meisten an...“, in: GZ, 24. 11. 1982. Vgl. außerdem die Leserbriefe: Noch nichts verstanden, in: GZ, 4./5. 12. 1982 und Nachhilfeunterricht, in: GZ, 21. 12. 1983.

25 Hiob, Idylle, S. 23.

26 Ebenda, S. 24. Vgl. ähnlich in: „Das geht Günzburg am meisten an...“, in: GZ, 24. 11. 1982.

27 Dieses und die folgenden Zitate: "Das geht Günzburg am meisten an...", in: GZ, 24. 11. 1982.

28 Zerrbilder, in: GZ, 27./28. 11. 1982.

29 Dieses und die folgenden Zitate: „Das geht Günzburg am meisten an...“, in: GZ, 24. 11. 1982.

30 Dieses und die folgenden Zitate: Zerrbilder, in: GZ, 27./28. 11. 1982. 
werte Nestbeschmutzung halten“. „Über Männer wie Josef Mengele und das Gewaltsystem, dem sie dienten, nachzudenken, hat nichts Anrüchiges“, schrieb der Günzburger Kommentator seinen Mitbürgern ins Stammbuch, „denn es geht primär nicht um Abrechnung, sondern um Lehren für die Zukunft."

Deshalb bedauerte es Roller auch, daß „in diesem Fall die Aufklärungsarbeit Leuten überlassen worden [ist], die mit purer Polemik zur Sache gehen und rasch bei der Hand sind mit Halb- und Unwahrheiten“. Hanne Hiob sei „offensichtlich mit jenem Zerrbild im Kopf angereist, das diverse Publikationen über Jahre hinweg gezeichnet haben“, von Günzburg als „Hort der Reaktion“ und „Hochburg des Nationalsozialismus“. Die Stadt müsse „mit der Tatsache leben, daß einer der meistgesuchten Judenmörder hier zu Hause war" und „noch viele Jahre hinweg mit der Familie in Kontakt blieb“. Gezielte „Provokation gegen die hier ansässige Familie“ mit der Hoffnung auf einen „zu propagandistischen Zwecken ausschlachtbaren Zwischenfall" helfe aber nicht weiter. Oberbürgermeister Dr. Rudolf Köppler (SPD) hatte sich bei seinem Besuch des Informationsstandes ähnlich geäußert: "Wenn's auch woanders gezeigt wird, ist das völlig legitim“31.

Letztendlich herausgekommen sei jedenfalls, so das Fazit Rollers, außer der Erkenntnis, daß es offensichtlich ein Wissensdefizit und Diskussionsbedarf gebe, „bei aller interessanten Information im Detail“ leider nur „ein hanebüchenes Gemisch ideologisch verbrämter Geschichtsklitterung“, das selbst jene, „die mit gutem Grund Nachholbedarf auf diesem Sektor konstatieren, irritiert" habe; ihr „schwieriges Geschäft vor Ort" sei dadurch nicht leichter geworden.

\section{Thematisierung der Vergangenheit: \\ $O B$ Köppler und "Der Aufbruch ins Verbängnis"}

Schon vor der Hiob’schen „Provokation“ gab es in Günzburg Indizien für ein steigendes Interesse an der Geschichte der Stadt im Nationalsozialismus. 32 Ähnlich wie „Holocaust" nicht der eigentliche Auslöser, sondern der Katalysator für ein neues Interesse der deutschen Gesellschaft am Nationalsozialismus gewesen war, scheint die „Provokation“ Hanne Hiobs in Günzburg ein Anlaß gewesen zu sein, der Stadtgeschichte während der NS-Zeit verstärkt öffentliche Beachtung zu schenken. So erschien etwa in der GZ ab diesem Zeitpunkt eine Artikelserie zu diesem Thema (meist aus der Feder Walter Rollers), beginnend mit einem Bericht über den Aufstieg der NSDAP in Günzburg anläßlich des 50. Jahrestages der Machtergreifung am 30. Januar 198333, und die örtliche Volkshochschule veranstaltete Vorträge zu einschlägigen Fragestellungen. ${ }^{34}$

31 „Das geht Günzburg am meisten an...“, in: GZ, 24. 11. 1982.

32 Vgl. z. B. der ganzseitige Bericht Walter Rollers in der GZ über die Nachforschungen Josef Baumeisters zum Schicksal des Stadtpfarrers Adam Birner: Der Tod eines Widerständlers, in: GZ, 16. 4. 1981. 1979 war außerdem die Dissertation Zdenek Zofkas erschienen, die auch Informationen zum Aufstieg des Nationalsozialismus in Günzburg bot. In welchem Umfang diese Studie in Günzburg rezipiert wurde, ist unbekannt; Zofka war später jedoch des öfteren Gesprächspartner der GZ oder Gast bei Diskussionsveranstaltungen.

$33 \mathrm{Vgl}$. Als Adolf Hitler kam, waren alle aus dem Häuschen; Günzburg marschierte voran... und Junge Leute gaben den Ton an, in: GZ, 29. 1. 1983.

34 So sprach z. B. am 1. Februar 1983 der Augsburger Historiker Dr. Walther Bernecker zu dem - sicherlich auch durch Hiobs antikapitalistischen Impetus angeregten - Thema „Kapitalis- 
Es bestand also Interesse, für das die Aktion Hanne Hiobs zwar nicht ursächlich war, das sie aber doch offengelegt und verstärkt hatte und auf das nun mit entsprechenden Angeboten reagiert wurde. Daneben hatten der Informationsstand und vor allem der Artikel in der KAZ (die am Stand verteilt worden war) den Günzburgern deutlich vor Augen geführt, welches Bild Außenstehende von ihrer Stadt hatten. Dieses Bild hatte sich seit den 1960er Jahren, in denen es hauptsächlich entstanden war, nicht geändert. Beide Faktoren dürften Oberbürgermeister Dr. Köppler (SPD) dazu bewogen haben, sich in einer Rede vor dem Stadtrat anläßlich einer Gedenkstunde zur Erinnerung an Machtergreifung und Gleichschaltung am 29. März 1983 mit den Problemen der deutschen Vergangenheitsbewältigung, der Geschichte Günzburgs während der NS-Zeit und dem durch den Fall Mengele geprägten Image der Stadt auseinanderzusetzen. ${ }^{35}$

Köppler begann seine Rede mit einer Distanzierung vom Begriff der „Kollektivschuld"36, wollte dies aber gerade nicht im Sinne des entgegengesetzten Extrems, also einer Kollektiventlastung, verstanden wissen. Vielmehr sah er die Gefahr, daß sich hinter der Verteidigung gegen solche ungerechtfertigten Pauschalvorwürfe gerade "diejenigen zu verstecken suchen, die individuelle Schuld auf sich geladen haben“. Zweifellos müßten die Deutschen „damit leben“, daß „im Namen unseres Volkes [...] Terror, Barbarei und grenzenloses Leid über die Menschheit gebracht wurden“. Wer sich dies bewußt mache, müsse „bestürzt" sein ob der "untauglichen" Aufrechnungsversuche "oder gar [des] Rechten[s] um Opferzahlen“. Die Deutschen täten sich schwer mit ihrer Vergangenheit, so Köppler, das Wort der Mitscherlichs von der "Unfähigkeit zu trauern" habe schon seine Richtigkeit. Die Deutschen "quälen sich" in der Konfrontation mit ihrer Vergangenheit, „sie werden schnell aggressiv, verfallen dann wieder in Wehleidigkeit, wollen am liebsten aussteigen (aus der Vergangenheit, Gegenwart, Zukunft)“.

Auch in Günzburg werde mittlerweile das "große Loch, das bislang in unserer ,Geschichte der Stadt Günzburg' gähnt "37, gefüllt, und für die Frage nach dem Aufstieg des Nationalsozialismus sei dies auch in "herausragender Weise“ geschehen, konstatierte Köppler und verwies unter anderem auf Zofkas Studie und die Artikel der GZ. Es lohne sich, „solche lokalen Bezüge aufzuspüren“, denn in der Erinnerung an "das dunkelste Kapitel in der Geschichte unseres Volkes" liege auch die Chance, „aus dem Schatten einer Geschichte zu treten, die auch den Namen von Städten verdunkelte“. Ähnlich wie Nürnberg als "Stadt der Reichsparteitage“ oder Dachau als Ort des Konzentrationslagers werde häufig auch Günzburg auf Josef Mengele reduziert. An dieser Stelle, so Köppler, „halte [ich] es für notwendig, [...] der Verhängung der Sippenhaftung gegenüber einer Familie und der Zuweisung von

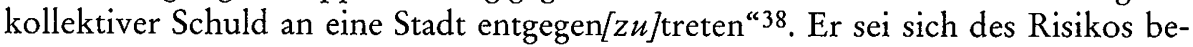

mus und Nationalsozialismus“. Die Ankündigung in der GZ versprach Überlegungen zu der Frage nach der „Unterstützung Hitlers durch die Industrie“; vgl. Historiker spricht, in: GZ, 29. 1. 1983.

35 Die VHS Günzburg veröffentlichte den Redetext später in ihrer Schriftenreihe „Günzburger Texte“. Köppler, Aufbruch. Vgl. außerdem die Zusammenfassung der Rede in der GZ:

Oberbürgermeister: Wir wollen nie vergessen, was einmal war, in: GZ, 30. 3. 1983.

${ }^{36}$ Dieses und die folgenden Zitate: Ebenda, S. 3.

37 Dieses und die folgenden Zitate: Ebenda, S. 4.

38 Dieses und die folgenden Zitate: Ebenda, S. 5. 
wußt und nehme es in Kauf, „daß hierin der Versuch des Abwiegelns gemutmaßt werden könnte“. Er sei aber der Meinung, „daß auch eine Stadtgemeinschaft Objektivität fordern kann, daß zur gerechten Beurteilung auch die Tatsachen gehören, die nicht unbedingt ins Konzept vorgefaßter Meinungen passen, und daß es mit Blick auf Günzburg nicht an Anlässen mangelt, die es gebieten, ein Zerrbild zu korrigieren".

„Nur gelegentlich, dann aber kraß und schlaglichtartig“ erführen die Günzburger, welches Bild von „unserer Stadt auch gezeichnet wird“. Der vor kurzem in der $\mathrm{KAZ}$ erschienene Artikel sei ein Beispiel, wie „unleugbare Tatsachen, aber auch Gerüchte und Vorurteile Selbstgerechter [...] zu einer Legende verwebt" würden, die aus der Stadt einen „Hort [...] Unbelehrbarer [mache], bei denen Strömungen aus Unwissenheit und Larmoyanz, verdrängter Schuld und nur schwach kaschierter Vergangenheitsverklärung zu einem trüben Gewässer zusammenfließen“. „Gerade weil Aufklärung zu begrüßen“ sei, müsse „mit Bedauern und Zorn festgestellt werden“, daß diese "polemische Mischung“ "dem Informationsanliegen den denkbar schlechtesten Dienst" erwiesen habe. Die "Abstrusitäten dieses mißratenen Versuchs der Vergangenheitsbewältigung" zeigten sich für ihn ganz persönlich darin, so Köppler, daß er „der willfährigen Sympathie zu einem Regime geziehen werde, dem mein Vater durch standrechtliche Erschießung aus politischen Gründen zum Opfer fiel“".

Im Anschluß daran bemühte sich der Oberbürgermeister, die zuvor geforderte, differenziertere Darstellung der Stadtgeschichte in der NS-Zeit zu leisten und in den größeren Kontext der Gesamtentwicklung des zeitgenössischen Deutschland einzuordnen. ${ }^{39}$ Der Frage, wie es zum Nationalsozialismus hatte kommen können, räumte er breiten Raum ein und betonte, „daß die NSDAP in Günzburg mit ihren Wahlergebnissen keinen Staat und erst recht nicht ,den Staat' hätte machen können"40. Eine Nazi-Hochburg sei Günzburg nicht gewesen, wie die Voten von 1930 bis 1933 zeigten, argumentierte Köppler zu Recht; an Hand der Persönlichkeiten Otto Geiselharts und Adam Birners und weiterer, weniger bekannter Fälle (vor allem Schutzhaftmeldungen im Günzburger Tagblatt/Günzburger Nationalzeitung) demonstrierte Köppler, daß die Wirklichkeit vielschichtiger war. ${ }^{41}$ Leider ging an dieser Stelle die eigentlich geforderte Differenzierung etwas verloren - daß die Nationalsozialisten, die die Schutzhaft vollstreckten, ebenfalls Günzburger waren, blieb weitgehend unerwähnt, ebenso wie die Geschichte Günzburgs im Nationalsozialismus in der Zeit nach 1933/34, die damals durchaus noch das von Köppler zu Beginn der Rede beklagte Loch in der Stadtgeschichte war.

Köppler verschwieg nicht, daß „die NSDAP in Günzburg [...] rege und stark [...] gewesen sei" ${ }^{42}$, konzentrierte sich aber in seiner Rede zunächst darauf, zu belegen, daß es in Günzburg auch „Gegnerschaft gab, die Verfolgung riskierte und erfahren mußte“. Natürlich mußte es dem Oberbürgermeister vor allem darum gehen, gegen ein schier übermächtiges Negativbild anzuargumentieren, das sich eben nicht auf die reale Geschichte der Stadt im Dritten Reich stützte, sondern auf die Namen Franz

39 Vgl. ebenda, S. 5-9.

40 Ebenda, S. 6.

41 Vgl. ebenda, S. 6 f.

42 Ebenda. 
Xaver Schwarz und vor allem Josef Mengele. Beide gehörten, so Köppler, natürlich auch „zur Geschichte unserer Stadt in jener Zeit" und seien diejenigen, die „am auffälligsten, weil bekanntesten, aus der dunklen Chronik herausragen " 43 . Sie seien jedoch "untereinander nicht vergleichbar", und nachdem Köppler zunächst über Schwarz und seine Verbindungen zu seiner Heimatstadt gesprochen hatte, wandte er sich Josef Mengele zu, dessen Namen „einen monströsen Schatten auf den Namen der Stadt" werfe. „Solange er in Günzburg lebte, und das war bis zum Jahr 1930, wurde über ihn nichts Nachteiliges bekannt", so Köppler. Er habe karitative Veranstaltungen des Roten Kreuzes mitgestaltet und sei „mit schwärmerischem Patriotismus" Mitglied des Großdeutschen Jugendbundes gewesen. 1930 habe er die Stadt verlassen, und danach „hat Günzburg Josef Mengele so gut wie aus den Augen verloren "44. Das, „was über den späteren Dr. Josef Mengele aktenkundig“ geworden sei, „kontrastiert bizarr“ mit dem, wie ihn die Günzburger bis 1930 kennengelernt hätten. Sicherlich sei Mengele „in seinem Denken und Handeln sehr stark durch die vaterländische Gesinnung und Haltung einiger Gymnasiallehrer geprägt" ${ }^{\text {" }}$ worden. Doch „wie hier jemand wurde, ob er nur so werden konnte “46, könne vielleicht „nie, und wenn, dann [nur] in einem Verfahren geklärt werden“".

„Materielle Gerechtigkeit“ oder „objektive Wahrheitsfindung“ sei schon in „einfacheren Verfahren kaum herstellbar“, es bleibe darum "nur die Herstellung von Wahrhaftigkeit: nichts soll vertuscht, nichts darf geschönt werden“. Die „nachgewachsenen Generationen" sollten um den Weg wissen, der ins Verhängnis geführt habe und gegen den "Günzburg ebensowenig wie jede andere Stadt gefeit" gewesen sei, denn „niemand kann garantieren, daß die Gefahr von Wiederholungen [...] für immer gebannt wäre". Auch in Günzburg seien solche Tendenzen leider wieder erkennbar. Er sei „über Zuspruch und Zulauf besorgt, den die Wiking-Jugend“, die den „germanischen Rassegedanken propagiere“, „an den Schulen unserer Stadt findet", ebenso wie über Zeitungsmeldungen aus benachbarten Städten, wo es zu „Drohungen und Terrorakten gegen [...] Türken“ gekommen sei. Deshalb sei es wichtig, die „Anatomie des Verhängnisses“ zu vermitteln und die „tatsächlichen Geschehnisse zum bewußten Bestandteil von Erfahrungen [...] unserer und künftiger Generationen zu machen“. Es könne nicht „darum gehen, rechthaberisch anzuprangern“. Das „eigentlich Anomale“ der „schlimmen Ära" sei ja gerade gewesen, "daß damals jede Stadt letztlich normal war“.

\section{Opfermythen: Josef Baumeister und „a' Stückle Hoimat"}

Nicht alle Deutschen waren von der neuen Erinnerungskonjunktur begeistert, die "Holocaust" ausgelöst hatte. Der seit der Nachkriegszeit im Selbstbild der Deutschen vorherrschende Mythos, sie selbst seien Opfer des Nationalsozialismus gewesen, war durch die emphatischen Bilder und durch die Darstellung der individu-

${ }^{43}$ Vgl. ebenda, S. 8 f.; dieses und die folgenden Zitate: Ebenda, S. 8.

44 Ebenda, S. 9. Mit den lokalhistorischen Recherchen für den Vortrag war übrigens Josef Baumeister betraut, den Köppler hier auch zitiert. Baumeister verharmloste auch später Mengeles Verbrechen in Auschwitz; die zu Mengeles Günzburger Zeit gemachten Angaben sind jedoch zuverlässig, wie auch diese Arbeit zeigen konnte.

45 Ebenda. Erneut zitiert Köppler Josef Baumeister:

46 Dieses und die folgenden Zitate: Ebenda. 
ellen Verfolgungsgeschichte einer jüdischen Familie in Frage gestellt worden. In einer Gegenreaktion revitalisierten besonders Angehörige der Generation der zwischen 1915 und 1935 Geborenen, die Generation der Soldaten des zweiten Weltkriegs, der Hitler-Jungen, Flak-Helfer und der Trümmerkinder ${ }^{47}$, den Mythos von den Deutschen als Opfern des Nationalsozialismus und forderte geradezu ultimativ einen Schlußstrich unter die Vergangenheit, die nach vierzig Jahren endlich ruhen müsse.

In Günzburg war dies nicht anders, wie die Reaktionen auf den Informationsstand Hanne Hiobs gezeigt hatten. „Was mit den Deutschen passiert ist, darüber redet keiner" 48 , hatte einer der Einwände gegen die Aktion gelautet, und auch andere Gegenstimmen hatten gezeigt, daß die Verdrängungs- und Abwehrmechanismen der 1950er und 1960er Jahre ihre Wirksamkeit noch nicht verloren hatten. ${ }^{49}$ Auch in so manchem Brief, den Günzburger oder in der Nähe von Günzburg lebende Leser in diesen Jahren an die Günzburger Zeitung richteten, wurde mehr oder weniger aggressiv auf den eigenen Opferstatus hingewiesen. ${ }^{50}$

Besonders augenfällig wurde dieser „boomerang effect" 51 auf nationaler Ebene in einem weiteren Fernsehfilm, der als direkte Antwort auf „Holocaust“ konzipiert war. Sofort nach dessen deutscher Erstausstrahlung begann der Filmemacher Edgar Reitz mit der Arbeit an dem Fernsehfilm „Heimat", der in fünfzehneinhalb Stunden (aufgeteilt in neun Episoden, die durchschnittlich jeweils rund neun Mio. Menschen sahen ${ }^{52}$ ) die Geschichte des Heimatdorfes des Autors vom Ende des Ersten Weltkrieges bis 1982 verfolgt. Jeder Hinweis auf das Schicksal der Juden blieb ausgespart, der Fernsehfilm zeigte ein idyllisches Dorf, das mit seinen aufrechten, ehrlichen Bürgern Opfer der Geschichte wurde. Die Frage nach den Opfern auf der anderen Seite, die Frage nach Auschwitz, blieb unbeantwortet. ${ }^{53}$

47 Vgl. zur Theorie der Alterskohorten: Marcuse, S. 290-296.

48 „Das geht Günzburg am meisten an...“, in: GZ, 24. 11. 1982.

49 Der Opfer-Mythos begann seine öffentliche Akzeptanz erst nach der berühmten Rede des Bundespräsidenten Richard von Weizsäcker am 8. Mai 1985 vor dem Deutschen Bundestag anläßlich des 40. Jahrestages des Kriegsendes zu verlieren. Der Bundespräsident unterschied darin klar zwischen den Deutschen als Opfern und den Deutschen als Tätern: „Die Initiative zum Krieg aber ging von Deutschland aus [...]. Die anderen Völker wurden zunächst Opfer eines von Deutschland ausgehenden Krieges, bevor wir selbst zu Opfern unseres eigenen Krieges wurden." Mit Blick auf die deutsche Teilung, für viele das Symbol deutschen Opfertums, hielt Weizsäcker fest, dies sei tatsächlich eine Nachkriegsentwicklung gewesen, „aber ohne den von Hitler begonnenen Krieg wäre sie nicht gekommen“. Ansprache des Bundespräsidenten Richard von Weizsäcker am 8. Mai 1985 im Plenarsaal des Deutschen Bundestages zum 40. Jahrestag der Beendigung des Zweiten Weltkrieges, in: Deutscher Bundestag Infothek, URL: http://www.bundestag.de/info/parlhist/dok26.html, Erstelldatum unbekannt, gesehen am 10.6.2002.

50 Als Beispiel sei ein Leserbrief eines Günzburgers zitiert, der in Reaktion auf die Demonstration Tuviah Friedmans (vgl. S. 161-164) geschrieben hatte: „Wenn 30 Jahre nach Kriegsende dem nicht Einhalt geboten wird, werden unsere Nachkommen noch in fünfzig oder hundert Jahren noch erpreßt werden. Ich selber bin Heimatvertriebener und habe alles verloren. Zwei Brüder von mir sind im Krieg gefallen. [...] Man soll die jetzigen Inhaber der Firma Mengele doch endlich in Ruhe lassen." Leserbrief: Verleumdung, in: GZ, 30. 1. 1985.

51 Marcuse, Legacies, S. 356.

52 "Holocaust" erreichte 10-13,4 Mio. Zuschauer pro Sendung. Damit waren „Holocaust“ und "Heimat" die erfolgreichsten Fernsehfilme der deutschen TV-Geschichte. Vgl. ebenda.

53 Timothy Garton Ash beantwortete die Frage „about the other side? What about Ausch- 
"A' Stückle Hoimat" 54 war auch Gegenstand einer Gedichtsammlung in schwäbischer Mundart, die der Günzburger Josef Baumeister, pensionierter Studiendirektor und Altphilologe, Ende 1983 kurz vor Weihnachten im Selbstverlag veröffentlichte. „Unverfänglich und gut" 55 höre sich der Titel an, so später die GZ, werde doch „allerorten [...] die Rückbesinnung aufs Heimatliche beschworen“. Die Texte verrieten „handwerkliches Können“, und auch sei Baumeister kein Dichter, der nur „auf ausgetretenen Pfaden“ wandele, „eine heile Welt beschwört und wehleidig über den Verlust jener Zeiten klagt, wo angeblich an Großmutters Herd noch alles in Ordnung war". In Baumeisters Büchlein findet sich viel Sozialkritisches, „Themen, die politisch umstritten sind und durchaus in Reflexionen über das Schicksal des heimatlichen Umfelds passen"; der Autor nahm sich in einem Abschnitt unter dem Titel „D'heitig Zeit" ${ }^{\circ 6}$ vieler Themen an, die Anfang und Mitte der 1980er Jahre, politisch aktuell und brisant waren, wie etwa der Nachrüstung ${ }^{57}$, der Umweltzerstörung 58 und des Baus neuer Atomkraftwerke ${ }^{59}$. Diese Gedichte seien ihm, „unterm Strich, in streckenweise hervorragender Manier aus der Feder geflossen “, so die GZ. In diesem Abschnitt befanden sich jedoch auch mehrere Gedichte, die das Buch letztlich zum „Skandal“" machten: Genannt sei etwa „Wo da na'gucksch"60, in dem Baumeister ein erschreckendes Maß an Xenophobie an den Tag legte ${ }^{61}$, und vor allem „An Dr. Josef Mengele" 62 , in dem Baumeister Josef Mengele als Opfer, ja Märtyrer stilisiert und ihn der Solidarität seiner Heimat versichert.

„Gewisse Kreise versuchten immer wieder, die Vorgänge um Dr. Josef Mengele in geradezu erpresserischer Weise durch gehässige, ja unsachliche Publikationen gegen die Angehörigen, die Stadt und letzten Endes gegen die Deutschen insgesamt zu mißbrauchen “63, so Baumeister in seinem Kommentar zu diesem Gedicht. „Dia wo nix wia Stoinr schmeißat / auf an $\mathrm{Ma}$ ', dear gfalla isch, bloß auf sei Verfehla weisat / send doch sell a o'guats Gmisch! / Denn dia Kerle zoigat daurat / bloß auf oin, dear onda leit" 64 , so der Anfang von Baumeisters Gedicht. Gemeint war damit nicht nur die Person Mengele, sondern der KZ-Arzt wurde zur Personifikation eines

witz", die sich ihm nach dem Film automatisch stelle, so: „Some things they [die Deutschen] remember in full color. Some in sepia. Others they prefer to forget." Zit. nach: Ebenda.

${ }^{54}$ [Ein Stückchen Heimat, hochdeutsch: S.K.]; Baumeister, Hoimat.

55 Dieses und die folgenden Zitate: Eine Hymne auf den „Todesengel“, in: GZ, 6. 4. 1984.

${ }^{56}$ [Die beutige Zeit].

57 "Keiat doch dia Waffa fut, legat d'Hoimat net en Schutt!“ Werft doch diese Waffen fort, legt die Heimat nicht in Schutt], in: Ebenda, S. 81.

58 "Neanads fendsch mea Koarabloama" [Nirgends findest Du mehr Kornblumen], in: Ebenda, S. 78.

59 „Dia Kerle vom Atom" [Die Kerle vom Atom], in: Ebenda, S. 80.

60 "Wo du auch hinschaust], in: Ebenda S. 96.

61 "Viel zviel hoimatfremde Gsichtr gahnt und standat om me rom. Wia lang daurats ond ma” brengt oin no em oigna Ländle om [...] Firchta muasch, daß Dir scho moara Turka gahnt da letschta Rest" [Viel zu viele heimatfremde Gesichter gehen und stehen um mich herum. Wie lange dauert es noch, und man bringt einen im eigenen Lande um [...] Fürchten mußt Du, daß Dir die Türken schon morgen den letzten Rest geben], ebenda.

62 In: Ebenda, S. 94 f. und S. 104 f. (Kommentar).

${ }^{63}$ Ebenda, S. 105. Baumeister schreibt auch, Mengele habe in Philosophie über Kant und Rosenberg promoviert, was natürlich falsch ist. Vgl. ebenda, S. 104.

${ }^{64}$ [Die, die nichts als Steine werfen, auf einen Mann, der gefallen ist, bloß auf sein Verfeblen weisen, sind doch immer ein ungutes Gemisch! Denn diese Kerle zeigen dauernd nur auf einen, der unten liegt], ebenda, S. 94, Hervorhebung im Original. 
Deutschland, dem immer noch und immer wieder seine Vergangenheit - ,in geradezu erpresserischer Weise" - vorgehalten wurde. Aus Baumeisters Sicht lag das aber nicht etwa an den Verbrechen, die Deutsche begangen hatten, sondern vor allem daran, daß Deutschland eben das Pech gehabt hatte, den Krieg zu verlieren: „Weil dr Kriag drneaba ganga, / klagt ma' Di ond's Volk heit a'."65

Überhaupt könne man Mengele ebensowenig wie allen anderen Deutschen einen Vorwurf machen; man habe damals halt sein Land geliebt: „Deitsche send an Deitschland ghanga, / so wia Du als jongr $\mathrm{Ma}^{3}$ "'66, und auch den Antisemitismus habe schließlich schon Luther gepredigt: „Hat et scho dr Luther böllat: / ,Zendat dSenagoga a'!' / Vom Kathedr hat ma's g'schellat, daß dr Jud bloß ,juda' ka / Vo Zegeinr ond Semita haba seltmaul ibral gseit: / Boide seiat Parasita, ibr dia koi Volk se frait." 67

Während also alten antisemitischen Klischees, wenn auch in Zitatform, viel Platz eingeräumt und ausgiebig die Opferrolle Deutschlands (der „Hoimat“) beschworen wurde, erwähnt Baumeister mit keinem Wort, wohin der nationalsozialistische Rassenantisemitismus führte. Über Auschwitz und die dortige Tätigkeit des Josef Mengele verliert Baumeister kein Wort. „Freile hasch a groaß Versaga / neibraucht in Dei Forscherei"68 ist die einzige Andeutung, die er in dieser Richtung macht: Mengele habe als Forscher halt Fehler gemacht, „doch ma ka’ De kaum verklaga, wo ganz Welt heit he ka' sei!"69 - In seinen Anmerkungen wird der Dichter noch deutlicher: Im Vergleich zur modernen Atombombenforschung seien Mengeles Taten „Entgleisungen [...], die sich geradezu harmlos ausnehmen“, und man solle „nach vierzig Jahren doch endlich aufhören, [darauf] in böser Absicht [...] hinzuweisen"70. "Viel hasch scho" die Jauhr her glitta: Ohne Hoimat bisch alloi! Zeit hoilt Wunda ond ka' kitta"71: Genug gelitten habe der heimatlose Flüchtling als

${ }^{65}$ [Weil der Krieg daneben gegangen ist, klagt man Dich und das Volk heute an], ebenda. Die Betonung einer Opferrolle Deutschlands findet sich auch in einigen weiteren Gedichten, so z. B. „Armes Deutschland“ (ebenda, S. 97): „Rengsrom lie'gat an de Rändr / volla Argwoah Häufa Ländr [...] Heit no semmer all Verbrechr [...] Fremde gahnt ein onsr Land, wo mir sell fascht kois mehr hand!" [Ringsherum liegen an den Rändern, voller Argwobn ein Haufen Länder [...] beute noch sind wir alle Verbrecher [...] Fremde kommen in unser Land, wo wir doch selbst fast keines mehr haben!]; ähnlich in "An jonge Deitsche" [An junge Deutscbe] (ebenda, S. 99): „Fir mi send dia, wo's deitsche Volk verdammat, viel mendr no wia dNazi allmitnand, weil die vom Deitsche heit no Zahlonga verlangat, au wenn se's, wia se sagat, vo Verbrecher hand!" [Für mich sind die, die das deutsche Volk verdammen, viel scblechter noch als alle Nazis miteinander, weil sie von den Deutschen heute noch Zablungen verlangen, auch wenn sie es, wie sie selbst sagen, von Verbrechern haben [nebmen, S.K.]]. Nicht unterschlagen werden soll auch die handschriftliche Widmung, die Baumeister der Ausgabe für die Universitätsbibliothek Augsburg vorangestellt hat: „Lange zertreten, wie Wildwuchs mißachtet, sahest Du allen Verleumdungen nach. Wer sich nicht wehrt und sich selber nicht achtet, hat keine Zukunft inmitten der Schmach!“.

${ }_{66}^{6}$ [Deutsche haben an Deutschland gehangen, so wie Du als junger Mann], ebenda, S. 94.

${ }^{67}$ [Hat nicht schon der Luther gebrüllt: , Zïndet die Synagogen an!' Vom Katheder bat man es verkü̈ndet, daß der Jude nur, juden' kann. Von Zigeunern und Semiten bat es damals überall gebeißen: Beide seien Parasiten, über die kein Volk sich freut], ebenda.

68 [Freilich hast Du ein großes Versagen in deine Forscherei hineingebracht], ebenda, S. 95.

69 [Doch man kann Dich kaum verklagen, wo die ganze Welt heute hin sein kann], ebenda.

70 Ebenda, S. 105.

71 [Viel hast Du wäbrend dieser Jabre schon gelitten: Obne Heimat bist du allein], ebenda, S. 95. 
Opfer derer, die nicht verzeihen könnten; wieder ist Mengele hier durchaus als Chiffre für Deutschland und die Heimat zu sehen. Dabei heile die Zeit schließlich die Wunden, und daheim mache man ihm keine Vorwürfe: „dHoimat wirft auf Di koin Stoi." 72

„Im Rathaus“, so die GZ, „hatte der Name Baumeister“ bis zu diesem Zeitpunkt „einen guten Klang"73. Der vorzeitig pensionierte Studiendirektor war im Jahr zuvor sogar mit historischen Nachforschungen für Köpplers Rede vor dem Stadtrat betraut worden. Kurz vor Weihnachten gab Baumeister zehn Exemplare seines Buches im Vorzimmer des Oberbürgermeisters $a b$ - offenbar in der Hoffnung, die Stadt werde mehrere Exemplare für Geschenkzwecke ordern. Nachdem er ein Exemplar durchgesehen hatte, so Köppler, habe er festgestellt, „daß die Stadt dieses Buch unmöglich [...] erwerben könnte" 74 . Acht der zehn Exemplare wurden an Baumeister zurückgesandt, die restlichen zwei behielt die Stadt als Belegexemplare. ${ }^{75} \mathrm{Als}$ die GZ sich Anfang April schließlich mit dem Gedichtband befaßte, zeigte sich der Oberbürgermeister gegenüber der Zeitung „entsetzt“, was er auch dem Autor gegenüber nicht verhehlte. ${ }^{76}$ Baumeister dagegen sah sich einer „Kampagne, mit einem unerhört starken Einsatz geführt", ausgesetzt, die ihn zwinge, "mit meinem Wissen an die Öffentlichkeit zu gehen"77. Was er mit seinem "Wissen" meinte, machte er in einem Schreiben an Köppler am 15. April 1984 deutlich: „Gerade durch mein intensives Studium mit [sic!] der Materie ist mir klar geworden, daß Mengele [...] nicht von ungefähr zur Symbolfigur deutschen Verbrechertums hochstilisiert wurde." Baumeister stilisierte Mengele zum unschuldigen Opfer: Für ihn lag „die größte Schweinerei in der Art, wie man diesen Menschen seit fast vierzig Jahren mit Kübel [sic!] voll Dreck in widerlichster Weise überschüttet"78. Mengele war für Baumeister „ein Märtyrer", den der Altphilologe in homerische Sphären entrückte: „Einst wird kommen der Tag“, so schrieb er offensichtlich in Anlehnung an die Ilias ${ }^{79}$, „an dem man das offen aussprechen wird - zur Freude seines Vaterlandes und seiner Heimatstadt." Mengeles Rehabilitierung widmete der Studiendirektor a. D. von nun an seine ganze Kraft. 80

72 [Die Heimat wirft auf Dich keinen Stein], ebenda, Hervorhebungen im Original.

73 Eine Hymne auf den "Todesengel“, in: GZ, 6. 4. 1984.

74 StAGz 130,1 (12), Spezialakt Josef Mengele, Bd. II, Schreiben Köpplers an Willy Schöllhorn betr. Artikel in der GZ, 30. 4. 1984.

75 Vgl. ebenda, Schreiben Baumeisters an Köppler betr. Gedichtband, 15. 2. 1984.

76 Vgl. ebenda.

77 Dieses und die folgenden Zitate: Ebenda, Schreiben Baumeisters an Köppler betr. Gedichtband, 15. 4. 1985.

78 Im Original unterstrichen.

79 „Einst wird kommen der Tag, da die heilige Ilios hinsinkt“. Hom. Il., 4, 164.

80 Dies belegen z. B. zahlreiche Schreiben Baumeisters an dic Stadt; auch Schreiben an andere Personen erhielt sie regelmäßig in Kopie. Vgl. StAGz 130,1 (12), Spezialakt Josef Mengele, Bd. II, Baumeister an Rolf Mengele betr. Artikel in Penthouse, 28. 7. 1986, Schreiben an den Günzburger Stadtrat betr. Glückwünsche zur Silberhochzeit und Mengele, 10.10. 1986; Schreiben an die Staatsanwaltschaft Frankfurt betr. Strafanzeige, 11. 3. 1986; Schreiben Baumeister an den Stadtrat betr. Publikation im Stern, 26. 8. 1986; Schreiben Baumeister an Ulrich Völklein betr. Artikel im Stern, 25. 3. 1985 und 8. 7. 1985; Schreiben Baumeister an die Staatsanwaltschaft Frankfurt betr. angebliche Verbrechen Mengeles, 24. 8. 1987; Schreiben Baumeisters an den Stadtrat und die Staatsanwaltschaft Frankfurt betr. Revision des tradierten Mengele-Bildes mit Anhang; Schreiben Baumeister an Köppler betr. NS-Verbrecher, 
Die Reaktionen des öffentlichen Günzburg waren eindeutig. Walter Roller sprach in der GZ von einer "gespenstische[n] Manipulation historischer Fakten" und einer „fatale $[n]$ Neigung, die Judenvernichtung zum leicht entschuldbaren Betriebsunfall herunterzuspielen“ und warf Baumeister vor, "neonazistischen Tendenzen auf verheerende Weise Vorschub" 81 zu leisten. Die Wirkung des Baumeister'schen Gedichtbandes blieb aber nicht auf Günzburg beschränkt. Anfang Mai, rund einen Monat nach Rollers Artikel im Lokalteil der GZ, äußerte sich Heinz Galinski, damaliger Vorsitzender der jüdischen Gemeinde in Berlin und späterer Vorsitzender des Zentralrats der Juden in Deutschland, zu dem Buch, das er „ungeheuerlich und unfaßbar" 82 nannte. Es füge „dem Ansehen der Stadt schwersten Schaden zu“. Galinski forderte OB Köppler außerdem dazu auf, sich von dem Buch zu distanzieren - was dieser ohnehin schon getan hatte und gern noch einmal wiederholte: „Das ganze ist entsetzlich. Baumeisters Äußerungen über Josef Mengele sind ungeheuerlich.“ Von weitergehenden Maßnahmen riet Köppler ab, denn es lohne nicht, „auf einen Mann wie Baumeister mit der Schrotflinte loszugehen“ und ihn ,auf diese Weise aufzuwerten“. Wieder plädierte Köppler dringend dafür, den einzelnen - in diesem Fall nicht Mengele selbst, sondern seinen Apologeten - nicht mit Günzburg zu identifizieren: „Baumeister ist ein unbelehrbarer Einzelgänger", den man nicht „zum Idol jenes Bodensatzes der Bevölkerung " machen dürfe, „der ähnlich denkt“.83

\section{1985: Die Kulmination des Falles Mengele}

\section{Der vierzigste Jahrestag der Befreiung von Auschwitz}

Die Ereignisse, die jene rasanten Entwicklungen auslösten, die schließlich im Juni 1985 zum Grab Mengeles in Embu führten, waren seit langem vorbereitet und auf das symbolträchtige Datum des vierzigsten Jahrestages der Befreiung des Konzentrationslagers Auschwitz hin ausgerichtet. Es sollte ein eindrucksvoller Versuch

Luther, Ausländerfeindlichkeit, 29.2 1988. Entsprechende Bestände finden sich auch in den Akten der Staatsanwaltschaft Frankfurt.

Vgl. außerdem den Leserbriefstreit in der GZ, der sich nach einer Veranstaltung der SPD mit dem Titel „Aus der Geschichte lernen - am Beispiel Mengele“ entspann. Bei der Veranstaltung hatten als Referenten $O B$ Köppler und Zdenek Zofka gesprochen, und im weiteren Verlauf hatte Baumeister für heftige Diskussionen gesorgt: Konfrontiert mit der Geschichte, in: GZ, 13. 5. 1985 sowie die Leserbriefe: Legendenbildung, in: GZ, 22. 5. 1985; Verbohrtheit, in: GZ, 23. 5. 1985; Bitte um Verzeihung, in: GZ, 28. 5. 1985; Voreingenommen, in: GZ, 1. 6. 1985; Ohne Moral und Ethik, in: GZ, 10.6. 1985; Kompetente Aussagen, in: Ebenda.

81 Eine Hymne auf den „Todesengel“, in: GZ, 6. 4. 1984.

82 Dieses und die folgenden Zitate: Galinski: Ein Schandfleck für Günzburg, in: GZ, 10. 5. 1984.

$83 \mathrm{Daß}$ es einen solchen Bodensatz auch im schwäbischen Raum gab, belegt der Brief eines Lesers aus Offingen, der seinen Kommentar zur Berichterstattung über den Artikel im Daily Express (vgl. S. 164ff.) mit deutlichen Reminiszenzen an antisemitische Weltverschwörungstheorien verband: „Zu allem Überfluß kamen auch noch die beiden Juden Friedman und Alster. [...] Warum läßt man 40 Jahre vergehen und beginnt dann die große Hetzkampagne? Ich glaube, daß diese Aktion über Mengele an das ganze deutsche Volk gerichtet ist. Das Ansehen der Deutschen ist in allen Ländern der Erde wieder gestiegen. Das paßt aber verschiedenen Leuten nicht in ihren Plan. Deshalb will man einen Mengele-Prozeß, um mit 
werden, „auf das Versagen der Regierungen bei der Ergreifung Mengeles aufmerksam zu machen" 84 und an ihr "Gewissen [...] zu rütteln" 85.1984 hatten sich unter der Bezeichnung C.A.N.D.L.E.S. (Children of Auschwitz Nazi Deadly Lab Experiments Survivors) annähernd einhundert Opfer Mengeles zusammengeschlossen, die unter großem internationalem Medieninteresse am 27. Januar, dem vierzigsten Jahrestag der Befreiung, das ehemalige Konzentrationslager Auschwitz besuchten. Die Rückkehr in das Vernichtungslager in Polen bildete den Auftakt zu einer Kampagne, die die Öffentlichkeit nachhaltig auf den Fall Mengele aufmerksam machen und die Regierungen und Behörden zu weiteren Fahndungsanstrengungen bewegen sollte. Schon der Auschwitz-Besuch hatte ein enormes, weltweites Medienecho ausgelöst. Nochmals übertroffen wurde die Medienwirkung aber von einer Veranstaltung, die vom 4. bis 6. Februar in der Gedenkstätte Yad Vashem in Jerusalem stattfand: Während des sogenannten Mengele-Tribunals, vor dem der Lagerarzt in Abwesenheit angeklagt wurde, schilderten über dreißig Überlebende ihre Leiden. Dem Tribunal gehörten unter anderem Telford Taylor (der amerikanische Chefankläger in Nürnberg), Gideon Hausner (der Ankläger Adolf Eichmanns) und Simon Wiesenthal an, der auch an der Organisation maßgeblich beteiligt gewesen war. Das Medieninteresse war gewaltig, drei Abende lang zeigten die Fernsehschirme in aller Welt Bilder von den teils schwer gezeichneten Opfern Mengeles, die ihr Leiden schilderten. ${ }^{86}$

Damit wuchs der öffentliche Druck vor allem in den USA. Dort hatten sich die Justizbehörden lange geweigert, eventuell vorhandenes Aktenmaterial im Zusammenhang mit Josef Mengele herauszugeben. Rabbi Marvin Hier, Leiter des Simon Wiesenthal-Zentrums in Los Angeles, hatte unter dem Freedom of Informations Act auf Herausgabe geklagt und Erfolg gehabt. Eineinhalb Jahre lang hatte Wiesenthal die Dokumente nicht veröffentlicht. ${ }^{87}$ Nun, unmittelbar vor dem AuschwitzBesuch der überlebenden Zwillinge, hatte Wiesenthal die Papiere publik gemacht ${ }^{88}$ : Eines von ihnen, das sogenannte "Gorby-Memorandum" 89 , enthielt den Hinweis, Mengele sei 1947 von den Amerikanern in Wien verhaftet worden. Die USA fürchteten nun einen zweiten Fall Barbie, und am 6. Februar 1985 wies der US-Generalstaatsanwalt das Justizministerium an, die Verwicklung der Vereinigten Staaten in den Fall Mengele zu untersuchen, alle Spuren zu verfolgen und auch mit ausländischen Ermittlungsbehörden zusammenzuarbeiten, um Mengele ausfindig zu machen. Die Ermittlungen führte das Office of Special Investigation (OSI) und der US-Marshals Service, alle anderen Dienste (wie NSA, CIA und FBI) wurden zu umfassender Kooperation verpflichtet. Es war eine der größten internationalen

dem Namen Mengele das deutsche Volk abermals in den Dreck zu ziehen. [...] Sucht endlich auch einmal nach denen, die Verbrechen am deutschen Volk begangen haben." Leserbrief: Sippenhaftung, in: GZ, 20. 3. 1985.

84 Posner/Ware, Mengele, S. 368.

85 Ebenda, S. 367.

86 Vgl. Artikel zum Thema z. B. in: SZ, 8. 2. 1985, DIE ZEIT, 14. 2. 1985 und Stern, 14. 2. 1985. Vgl. außerdem: Posner/Ware, Mengele, S. 367 f.; Kubica, Mengele, S. 429, und Lagnando/ Dekel, Zwillinge, S. 233-236.

87 Vgl. Posner/Ware, Mengele, S. 368.

88 Angeblich neue Dokumente über Mengele, in: SZ, 25. 1. 1985.

89 Kopie in: In the Matter of Josef Mengele 2, S. 86. 
Suchaktionen in der Geschichte der Vereinigten Staaten. Zudem entschloß sich der amerikanische Kongreß, einen Untersuchungsausschuß zu bilden, der rund zwei Wochen später seine Arbeit aufnahm. ${ }^{90}$

Von nun an entwickelte der Fall Mengele eine ungeahnte Dynamik. Die weltweit ausgesetzten Belohnungen vervielfachten sich auf die enorme Summe von umgerechnet zehn Millionen DM. In den wenigen Tagen rund um den 27. Januar 1985 war im Fall Mengele zumindest eine entscheidende Entwicklung eingeleitet, wenn nicht sogar ein Wendepunkt erreicht worden: Das internationale Interesse war groß wie nie zuvor, und die USA, Israel und Deutschland beschlossen unter dem wachsenden Druck der öffentlichen Meinung, ihre Bemühungen deutlich zu intensivieren und endlich auch zu koordinieren. Wiederum waren es "Skandale" gewesen, die, diesmal bewußt und wirkungsvoll eingesetzt und mit einem symbolträchtigen Datum verknüpft, das gesellschaftliche Interesse erregt und die öffentliche Meinung mobilisiert hatten. Die Hoffnung, die beteiligten Regierungen unter Zugzwang zu setzen und sie de facto zu zwingen, der Jagd nach Mengele höhere Priorität zu gewähren, war besonders im Fall der USA gelungen; ohne Übertreibung wird man sagen können, daß dies den weiteren Verlauf der Ereignisse entscheidend beeinflußt hat. Natürlich blieben diese Entwicklungen auch für Günzburg nicht ohne Folgen.

\section{Günzburg im Mittelpunkt des Interesses}

Tuviah Friedman ${ }^{91}$ - Forderungen an die Stadt

Der erste „Skandal“, der Günzburg mit dem Fall Mengele konfrontierte, war noch keine Folge der Ereignisse um den vierzigsten Jahrestag der Befreiung des Konzentrationslagers Auschwitz, vielmehr gehört er unter strukturellen Gesichtspunkten dazu - zumindest verfolgte sein Urheber die gleichen Ziele wie Simon Wiesenthal und die Opfer Mengeles, ohne dabei auch nur annähernd vergleichbaren Erfolg zu haben. Am 17. Januar 1985 setzte der Pressereferent der Deutschen Botschaft in Tel Aviv, Hans-Peter Kaul, Oberbürgermeister Köppler davon in Kenntnis, daß der „Israeli Tuvia Friedmann beabsichtigt, voraussichtlich am 27.01. 1985 in Günzburg zu demonstrieren "92. Er habe „,in einer Pressekonferenz in Tel Aviv, die viel Aufsehen erregte, erklärt, daß er am 40. Jahrestag der Befreiung des KZ Auschwitz vor dem Geburtshaus des Dr. Josef Mengele und vor dem Günzburger Rathaus zu demonstrieren gedenke. Außerdem wolle er mit dem Bürgermeister sprechen". Die Demonstration Friedmans, der entgegen seiner ursprünglichen Planung bereits am 23. Januar in Günzburg erschien, wurde für die Stadt zu einem Prolog dessen, was sie in den kommenden Monaten erwartete.

Tuviah Friedman war, ähnlich wie Simon Wiesenthal, ein Nazi-Jäger. Beide lernten sich nach dem Krieg in Wien kennen, wo sie für die „Brichah“ arbeiteten, eine Geheimoperation, die die zu diesem Zeitpunkt noch illegale Auswanderung von

$90 \mathrm{Vgl}$. Searching for Dr. Josef Mengele und Zuroff, Beruf, S. 125-143.

91 Der Name Friedman findet sich in den unterschiedlichsten Schreibweisen, wie etwa Tuvia/ Tuviah/Tovyya/Tobias Friedmann/Friedman. In Zitaten werde ich die jeweilige Schreibweise beibehalten, ansonsten wähle ich „Tuviah Friedman“.

92 Dieses und das folgende Zitat: StAGz 130,1 (12), Spezialakt Josef Mengele, Bd. II, Aktennotiz betr. Telefonanruf, 18. 1. 1985. 
Juden nach Palästina organisierte. Friedman blieb bis 1952 in Wien, wo er ein kleines Dokumentationszentrum für NS-Verbrechen betrieb - vergleichbar der Linzer Institution Wiesenthals. Später ging Friedman nach Israel, wo er nach einer kurzen Tätigkeit für die Gedenkstätte Yad Vashem in Haifa ein weiteres Dokumentationszentrum errichtete. Friedman erreichte nie den Bekanntheitsgrad, den Einfluß und die Medienwirkung seines früheren Freundes und späteren Konkurrenten Simon Wiesenthal, mit dem er sich über die Frage zerstritten hatte, wer von beiden wie viel zur Ergreifung Adolf Eichmanns beigetragen hatte. ${ }^{93}$

Die Beschreibung Friedmans durch den Pressereferenten Kaul fiel wenig schmeichelhaft aus. Er sei „selbst NS-Opfer und kehre dies seit vier Jahrzehnten demonstrativ heraus. Er ist keine seriöse Erscheinung und gilt als Lobbyist", außerdem müsse er „eher als ,selbsternannter Kopfjäger" angesehen werden" 94 . Friedman sei „sowohl dem Auswärtigen Amt als auch dem Bundesjustizministerium sowie der Deutschen Botschaft bekannt "95. Allgemein gelte er auch in Israel „als publizitätssüchtig und außerordentlich medienerfahren“ und verfüge über „ein hohes Schadenspotential“96. Nachdem Köppler dem Pressesprecher unter Verweis auf seine Rede vor dem Stadtrat 1983 mitgeteilt hatte, die Stadt habe „unmißverständlich zum Phänomen des Nationalsozialismus [...] Stellung " genommen, bat dieser um eine Übermittlung des Kurztextes per Telex, „um mit Herrn Friedmann noch vor dessen Abreise darüber sprechen zu können, daß dieser nicht in eine ,Nazi-Hochburg komme" 97 .

In einem weiteren Telefonat unterrichtete Kaul den Oberbürgermeister vom Verlauf des Gesprächs mit Friedman. Er habe diesem mitgeteilt, daß Köppler bereit sei, „ihn möglichst bald zu sehen und zu empfangen“98. Friedman habe sein Eintreffen für den 24. Januar angekündigt und mitgeteilt, worüber er mit dem Stadtoberhaupt sprechen wolle. Es gehe ihm um die Frage, „was kann die Stadt dazu beitragen, daß Mengele sich stellt?" und eine Zusage, „daß Mengele nicht auf dem Günzburger Friedhof begraben wird, falls er ohne Prozeß sterben sollte" 99 . Kaul habe extra darauf hingewiesen, „mit welchen emotionalen und publizitätsträchtigen Anliegen Herr Friedmann nach Günzburg kommen wird"100.

Tuviah Friedman kam bereits einen Tag früher als angekündigt nach Günzburg, am 23. Januar 1985.101 Deswegen wurden er und sein Begleiter, Josef Alster, vor-

${ }^{93}$ Vgl. Pick, Wiesenthal, S. 95 und 105. Pick spricht von Friedmans „little-known Documentation Center" (S. 105).

94 StAGz 130,1 (12), Bd. II, Aktennotiz betr. Telefonanrufs des Pressesprechers der Deutschen Botschaft in Israel, 17. 1. 1985.

95 Ebenda.

96 Ebenda. Vgl. außerdem StAGz 130,1 (12), Bd. II, Telex des Deutschen Botschafters Hansen betr. Friedman, 17. 1. 1985, bei dem es sich um einen für das Auswärtige Amt bestimmten Bericht zu handeln scheint, der der Stadt Günzburg in Kopie zugeleitet wurde.

${ }_{77}$ StAGz 130,1 (12), Bd. II, Aktennotiz betr. Telefonanrufs des Pressesprechers der Deutschen Botschaft in Israel, 17. 1. 1985.

${ }^{98}$ StAGz 130,1 (12), Bd. II., Gesprächsnotiz betr. Telefonanrufs dcs Pressesprechers der Deutschen Botschaft in Israel, 18. 1. 1985.

${ }_{99}$ Ebenda.

100 Ebenda.

101 Kaul hatte dies in einem weiteren Telefongespräch angekündigt. Vgl. StAGz 130,1 (12), Bd. II., Gesprächsnotiz betr. Telefonanruf des Pressesprechers der Deutschen Botschaft in Israel, 21. 1. 1985. Vgl. zum Verlauf des Besuchs Friedmans: StAGz 130,1 (12), Bd. II, 
mittags zunächst von einem Beamten der Stadtverwaltung im Amtszimmer des Oberbürgermeisters, der auf Grund der kurzfristigen Terminänderung zunächst verhindert war, empfangen. Außerdem waren verschiedene Pressevertreter anwesend, so die GZ, die Presseagenturen AP und Reuter und die ARD. ${ }^{102}$ Zunächst, so das Gesprächsprotokoll, habe Friedman über die Person Mengeles, seine Verbrechen und seinen vermuteten momentanen Aufenthaltsort gesprochen, bevor er feststellte, daß sich dieser „nur deshalb noch in Freiheit befindet, weil seine Verfolgung [...] nicht mit dem nötigen Nachdruck betrieben wird"103. Natürlich gab es auch Anklänge an den - mittlerweile beinahe klassisch zu nennenden - Mengele-Mythos: Der „Eichmann Nummer zwei“ werde vom „Präsidenten von Paraguay, der Bundesregierung und der amerikanischen Regierung gedeckt", so war sich Friedman sicher, und er sei ein „Multimillionär“, der in Freiheit „frißt und den besten Champagner trinkt"104.

Friedman behauptete, er habe „als ,Nazijäger 3000 Verbrecher hinter Gitter gebracht“ und hoffe nun, Mengele „zwingen zu können, daß er sich selbst [...] stellt"105. Dann erläuterte er, wie er den Druck auf Mengele erhöhen wolle: „Diese sündige Stadt" sei „wenigstens moralisch mitverantwortlich an den Untaten Dr. Mengeles. Sie soll deshalb ,nicht feig sein' und [...] ,ein Geschrei erheben' [...], um Dr. Mengele doch noch dem Richter zuzuführen."106 Die Stadt solle anregen, das Kopfgeld von 50000 DM auf eine Million anzuheben, sich selbst daran beteiligen „(vielleicht 100000 DM)“ und die „Familie Mengele dazu bewegen, ebenfalls einen finanziellen Beitrag zu leisten“. Die Stadt „soll ,keine Angst vor der Firma Mengele haben' und auf sie Druck ausüben", daß sich Dr. Mengele stellt". Besonderen Erfolg versprach sich Friedman offenbar von einer „Regelung, daß die Familie nur dann Dr. Mengele auf dem Günzburger Friedhof begraben darf, wenn er sich [...] freiwillig stellt". Er glaube, daß Mengele als „frommer Katholik" 107 sich von der Drohung unter Druck setzen lasse, ansonsten "niemals in seinem Heimatboden begraben“ zu werden. Nur so könne sich Günzburg von der „Schande“ 108 befreien, denn „wenn sich in diesem Jahr Mengele nicht findet, dann wird es dieser Stadt gehen wie mit Sodom und Gomorrha“"109. Schließlich sei „sicher, daß es in Günzburg Leute gibt, die wissen, wo Dr. Mengele ist und ihn finanziell unterstützen" 110 .

Aktennotiz betr. Besuch Fricdmans und Alsters, 23. 1. 1985 und Sitzungs-BesprechungsVorlage betr. Besuch Friedmans und Alsters, 23. 1. 1985; Nazi-Jäger auf der Suche nach Mengele, in: GZ, 24. 1. 1985; Dieser Stadt geht's noch wie Sodom und Gomorrha ..., in: GZ, 24. 1. 1985 und Stadt soll Mengele jetzt jagen helfen, in: GZ, 24. 1. 1985.

102 Die überregionalen Presseorgane hatte offenbar Friedman verständigt. Vgl. StAGz 130,1 (12), Bd. II, Sitzungs-Besprechungs-Vorlage betr. Besuch Friedmans und Alsters, 23. 1. 1985.

103 StAGz 130,1 (12), Bd. II, Aktennotiz betr. Besuch Friedmans und Alsters, 23. 1. 1985.

104 Dieser Stadt geht's noch wie Sodom und Gomorrha ..., in: GZ, 24. 1. 1985.

105 Ebenda.

106 Dieses und die folgenden Zitate: StAGz 130,1 (12), Bd. II, Aktennotiz betr. Besuch Friedmans und Alsters, 23.1. 1985. Vgl. außerdem: Stadt soll Mengele jetzt jagen helfen, in: GZ, 24. 1. 1985.

107 Stadt soll Mengele jetzt jagen helfen, in: GZ, 24. 1. 1985.

108 Ebenda.

109 Dieser Stadt geht's noch wie Sodom und Gomorrha..., in: GZ, 24. 1. 1985.

110 StAGz 130,1 (12), Bd. II, Aktennotiz betr. Besuch Friedmans und Alsters, 23. 1. 1985. 
Nach dem Gespräch im Rathaus demonstrierten Friedman und Alster mittags auf dem Günzburger Marktplatz, wo sie allerdings "nicht viel Aufsehen erregten"111. Dabei hatten Friedman und Alster „ganz ähnliche Reaktionen“ ausgelöst „wie vor wenigen Jahren Hanne Hiob"112. Viele hätten sich verteidigt, daß „doch die Günzburger nichts dafür [können], daß Mengele hier geboren ist" oder gefragt, was sie „denn jetzt noch von dem" wollten, „er ist doch schon über 70 Jahre alt"113. Anschließend versuchten die Israelis, auch auf dem Werksgelände der Firma Mengele zu demonstrieren, wurden aber von der Werksleitung des Betriebsgeländes verwiesen. Dieter und Karl-Heinz Mengele lehnten ein Gespräch ab. Statt dessen sprachen Friedman und Alster mit Landrat Simnacher, bevor sie ins Rathaus zurückkehrten, um doch noch mit Bürgermeister Köppler zusammenzutreffen - sie hatten vorher schon positiv vermerkt, "daß er sich nicht drückt" ${ }^{\prime 14}$. Viel mehr erreichten die beiden allerdings nicht: Der Günzburger Bürgermeister machte seinen Gästen in aller Deutlichkeit klar, daß es seiner Ansicht nach „das Bemühen um historische Gerechtigkeit verbiete [...], den Namen der Stadt untrennbar mit den Nazi-Verbrechen Mengeles zu verquicken"115. Auf eine Bestattung im Familiengrab habe Josef Mengele ohnehin keinen Anspruch ${ }^{116}$, und weitergehende Maßnahmen lehnte Köppler ebenfalls ab, denn „bei uns hat die öffentliche Distanzierung von Mengele stattgefunden"117, wie er unter Bezugnahme auf seine Rede vor dem Stadtrat von 1983 erklärte. Die Verteidigungslinie Köpplers gegen die in der Tat „rechtlich wie auch inhaltlich [...] abwegig/en]" ${ }^{\prime 18}$ Forderungen Friedmans war erneut der Appell, die Stadt nicht in einen Topf zu werfen mit den Verbrechen des Josef Mengele.

\section{Daily Express - Der Günzburg-Mythos als Boulevard-Schlagzeile}

Nachdem der Fall Mengele nach dem 27. Januar in den Focus der internationalen Öffentlichkeit gerückt war, dauerte es nicht lange, bis die Medien auch die Geburtsstadt des KZ-Arztes als lohnendes Ziel (wieder)entdeckten. In den Wochen nach dem Mengele-Tribunal erlebte Günzburg einen bis dahin noch nie dagewesenen Presserummel. In der Stadt hielten sich eine Vielzahl von Journalisten auf ${ }^{119}$, darunter Vertreter der amerikanischen Fernsehsender CBS und ABC oder der englischen Tageszeitung Daily Mail. ${ }^{120}$ Die meisten wandten sich bei ihren Nachforschungen auch an Oberbürgermeister Köppler, der befürchtete, , es könnte ein tendenziell gefärbtes Bild sein“121, das von der Stadt entstehe. Aus den „bohrenden und insistierenden" Fragen könne man ersehen, so der OB, daß die Reporter vor allem dem in

111 Nazi-Jäger auf der Suche nach Mengele, in: GZ, 24. 1. 1985.

112 Dieser Stadt geht's noch wie Sodom und Gomorrha..., in: GZ, 24. 1. 1985.

113 Ebenda.

114 StAGz 130,1 (12), Bd. II, Aktennotiz betr. Besuch Friedmans und Alsters, 23. 1. 1985.

115 Stadt soll Mengele jetzt jagen helfen, in: GZ, 24. 1. 1985.

116 Vgl. StAGz 130,1 (12), Bd. II, Aktennotiz betr. Besuch Friedmans und Alsters, 23. 1. 1985. Dies hatte der Leiter des Rechtsamtes bereits am Vormittag erklärt.

117 Stadt soll Mengele jetzt jagen helfen, in: GZ, 24. 1. 1985.

118 So ein hinsichtlich eines verbreiteten Desinteresses an der NS-Vergangenheit in Günzburg durchaus kritischer Kommentar im Mantelteil (Augsburger Allgemeine) der GZ: Offene Wunde, in: GZ, 26./27. 1. 1985.

119 Vgl. Dunkle Story eines Massenblattes, in: GZ, 23./24. 2. 1985.

120 Vgl. Köppler kämpft um den guten Ruf Günzburgs, in: GZ, 23./24. 2. 1985.

121 Ebenda. 
den 1960er Jahren entstandenen Bild von Günzburg nachspürten, „für das es vor zwanzig Jahren sogar eine offizielle Bestätigung der Oberstaatsanwaltschaft in Frankfurt gegeben"122 habe. Wie recht er mit seiner Befürchtung hatte, demonstrierten die Reporter der Londoner Zeitung Daily Express, die sich gar nicht erst um einen Gesprächstermin mit dem Bürgermeister bemüht hatten. Am 18. Februar 1985 berichtete das Boulevardblatt dann „exclusive“ 123 unter dem Titel „The town where Dr Death still casts his evil shadow" aus der Heimatstadt des KZ-Arztes.

Sie sehe eigentlich aus wie jede andere Kleinstadt in diesem Teil Deutschlands, so der Beginn des Artikels, aber: „Gunzburg is different. It is a town with a dark secret. Overshadowed by the name of a man whose exploits [...] have earned him a secure place in the annals of evil." Danach folgt auf zwei großformatigen Zeitungsseiten eine wilde Melange der etablierten Zutaten des Mengele-Mythos, vermengt mit einigen, Ortskenntnis vorspiegelnden Erfindungen - so wollen die Reporter an der Stelle, an der Hitler $1932^{124}$ auf dem Marktplatz gestanden habe, einen Gedenkstein entdeckt haben: „His visit was to praise the Mengele family's efforts.“ Mehr als die Hälfte der erwachsenen Bevölkerung arbeite in der Landmaschinenfabrik, viele der übrigen hingen anderweitig von den Mengeles ab.

Nach einigen kurzen Sätzen zu Mengeles Biographie und seiner Tätigkeit in Auschwitz beschrieben die beiden Autoren, wie sehr die Familie Mengele die Stadt im Griff habe. ${ }^{125}$ Anschließend erfuhren die Leser, daß Karl-Heinz Mengele es auf die ohnehin wenigen Juden in Günzburg abgesehen habe und Leute, die mit ihnen verkehrten, nicht mehr zu seinen Freunden zähle. „The Mengeles know everything that happens here. Their story is that Josef is dead and they discourage every conversation about it", so zitierten die Autoren eine namentlich genannte Zeugin, die einen Beauty-Salon betreibe und auch zwei von Josefs Nichten zu ihren Kundinnen zähle. Diese Zeugin kommt mehrfach zu Wort - und hat ihr Gespräch mit den beiden Reportern ganz anders in Erinnerung: Sie sei in der vergangenen Woche von zwei Engländern angesprochen worden, sagte sie später der GZ. Wohl, um ihren Namen zu erfahren, hätten diese ihr gegenüber vorgegeben, „auf Geschäftsreise [...] zu sein und ihr von ihrer frisch verheirateten englischen Bekannten Grüße ausrichten zu wollen"126. Folglich „fiel sie aus allen Wolken"127, als sie von den Aussagen erfuhr, die sie angeblich den Reportern gegenüber gemacht haben sollte. Von ähnlichem Wert ist eine Geschichte, die die Engländer mit einem - diesmal anonym bleibenden - Informanten in einem Café erlebt haben wollten: „A whispered conversation [...] was brought to an abrupt end when a smartly dressed woman arrived at

122 Ebenda.

123 Dieses und die folgenden Zitate: The town where Dr Death still casts his evil shadow, in: Daily Express, 18. 2. 1985.

124 Das Foto, das daneben abgedruckt ist, zeigt den Hitler-Besuch von 1930.

125 Ihre Aktivitäten durchdrängen alle Lebensbereiche der Stadt, es folgt eine lange Auflistung: Von einer Amtszeit Karl-Heinz' als Präsident des Rotary-Club ist die Rede, vom HandballTeam, das vom Sponsoring seines Präsidenten (ebenfalls Karl-Heinz Mengele) profitiere, vom Kindergarten, den die Mengeles gespendet hätten. Mehrere Familienmitglieder hätten im Stadtrat die Politik beeinflußt [tatsächlich war nur Karl Mengele Sr. im Stadtrat], und sogar das Familiengrab sei das größte auf dem Friedhof, immer mit frischem Lorbeer bekränzt und direkt neben der Kapellentür. Vgl. ebenda.

126 Dunkle Story eines Massenblattes, in: GZ, 23./24. 2. 1985.

127 Ebenda. 
the next table. Our informant took my notebook and wrote in it: ,She is the wife of the mayor. They are close to the Mengeles. "Then got up and left." 128

Begebenheiten wurden in dem Artikel verkürzt wiedergegeben, um ins Schema zu passen, so zum Beispiel die nur wenige Wochen zurückliegende Demonstration Friedmans. „Most of the town just walked by with their heads turned away. But a few were openly hostile. Finally, the Mengeles' security men had the demonstrators removed from the factory precinct." Daß Friedman sowohl mit dem Oberbürgermeister als auch mit dem Landrat gesprochen hatte, blieb unerwähnt. Viele Aussagen wurden Günzburgern nach dem oben dargestellten Schema in den Mund gelegt - etwa einem Reporter der Lokalzeitung, von der es eigentlich heißt, sie müsse sich linientreu („had to fall in line“) verhalten, denn natürlich sei auch sie abhängig von den Mengeles. Was hätte den Redakteur dann - der immanenten Logik der Behauptungen des Daily Express folgend - dazu bewegen sollen, durch die Kolportage einer ganzen Reihe von Gerüchten und Legenden und durch Mengele-kritische Aussagen gegenüber der internationalen Presse seine Position und die seiner Zeitung aufs Spiel zu setzen?

Die Genese vieler Legenden, die die Reporter aufgriffen, läßt sich innerhalb des Mengele-Mythos bis zu ihrem Ursprung zurückverfolgen. Sie stammen aus dem großen Fundus, der sich seit den 1960er Jahren gebildet hatte und aus dem sich die Autoren hemmungslos bedienten ${ }^{129}$; allerdings gab es bei der Adaption in die eigene Story immer wieder kleine Schönheitsfehler: So verwechseln die Autoren im Falle der Legende, Josef Mengele habe 1959 die Beerdigung seines Vaters besucht, den Anlaß und sprechen von einem angeblichen Besuch anläßlich der Beerdigung Alois' Mengeles, die sie dann obendrein noch falsch, nämlich ins Jahr 1972 statt 1974, datieren. Im gleichen Zusammenhang gab es das Gerücht, Mengele sei anläßlich des angeblichen Besuchs 1959 im Kloster der Englischen Fräulein versteckt worden daraus machen die Daily Express-Reporter Mengeles Nachkriegsversteck und aus den Nonnen wurden Mönche: „He definitely returned zu Gunzburg after the war, living with monks in a nearby community until his fathers death in $1951[!]^{*}$.

Verwundern allerdings kann dies nicht. Als die beiden Reporter nach Günzburg kamen, befanden sie sich auf der Heimreise von der Berichterstattung über eine Gruppe tödlich verunglückter Militärmusiker und verließen die Stadt bereits am nächsten Tag wieder. Sie hielten sich also gerade zwei Tage und eine Nacht in der Stadt auf ${ }^{130}$, offensichtlich gerade lange genug, um sich mit Namen und oberflächlicher Ortskenntnis auszustatten, um später profunde Recherchen vorspiegeln zu können. Dieses Gerüst aus realen Namen und Orten betteten die Journalisten in den Mengele-Mythos und füllten es dann mit Gerüchten und Legenden aus der Schatzkiste des seit Jahrzehnten etablierten Günzburg-Mythos; gewissermaßen als Krönung mischten sie zuletzt noch einige Episoden bei, die gut genug erfunden schienen, um die Klischees zu bestätigen.

${ }^{128}$ Dieses und die folgenden Zitate: The town where Dr Death still casts his evil shadow, in: Daily Express, 18. 2. 1985.

129 Die vielen Legenden, die in dem Artikel kolportiert werden und nicht direkt im Zusammenhang mit Günzburg stehen, sollen hier nicht detailliert wiedergegeben werden. Jedenfalls folgten die Autoren dem aktuellen Mengele-Mainstream und waren von der Schlüsselrolle Alfredo Stroessners und des amerikanischen Geheimdienstes überzeugt.

130 Vgl. Dunkle Story eines Massenblattes, in: GZ, 23./24. 2. 1985. 


\section{Petra Kelly - Politische Instrumentalisierung}

Die von den Daily Express-Reportern gleich mehrfach in falschem Kontext verwendete Legende, Josef Mengele habe 1959 das Begräbnis seines Vaters besucht, machte wenig später erneut Schlagzeilen, als Petra Kelly, die in Günzburg geborene Bundestagsabgeordnete der Grünen, die Geschichte (wieder)entdeckte. Die Politikerin hatte in Günzburg als Kind das Institut der Englischen Fräulein besucht, die Schule jenes Klosters also, von dem erstmals Simon Wiesenthal behauptet hatte, daß dort Josef Mengele anläßlich seines Besuchs 1959 übernachtet habe. ${ }^{131}$

In einer parlamentarischen Anfrage befaßte Kelly am 14. März 1985 die Bundesregierung mit dem Thema. Die Interpellation verknüpfte vier Anfragen zum Fall Josef Mengele ${ }^{132}$ mit dreien zum geplanten, politisch zu diesem Zeitpunkt sehr umstrittenen Besuch des paraguayanischen Diktators Alfredo Stroessner in der Bundesrepublik ${ }^{133}$. Stroessner wurde zu diesem Zeitpunkt immer noch vielfach verdächtigt, er verstecke den KZ-Arzt. Durch die Verknüpfung beider Themen sollte offensichtlich die Tatsache, daß die Bundesregierung plante, den angeblichen Mengele-Protektor zu empfangen, in Kontrast gesetzt werden mit den Versäumnissen Deutschlands bei der Suche nach Josef Mengele, stellvertretend für die nachlässige Verfolgung von NS-Tätern.

Einige Tage nach der Interpellation wandte sich Kelly mit der Schlagzeilen versprechenden Story an die Medien, drei Nonnen hätten ihr anläßlich eines Besuchs berichtet, der Massenmörder Mengele sei in seiner Heimatstadt im Jahr 1959 vier bis fünf Tage in einem Frauenkloster versteckt worden. ${ }^{134}$ Später präzisierte sie gegenüber der GZ, es habe sich dabei um „drei sehr persönlich geführte Gespräche gehandelt" 135 , die, wie sie später selbst in einem Leserbrief schrieb, „drei oder vier Jahre“ 136 zurücklägen. In einer einige Wochen später durch die Staatsanwaltschaft veranlaßten Vernehmung zeigte sich die tatsächliche Substanz der Geschichte: Die

131 Soweit ich dies überblicken kann, wurde dieses Detail der Legende erstmals 1967 in Simon Wiesenthals Buch „Doch die Mörder leben“ erwähnt. Vgl. Wiesenthal, Mörder, S. 204.

132 „1. Hat die Bundesregierung Auskünfte über einen Besuch von Joseph [sic!] Mengele bei der Beerdigung seines Vaters in Günzburg/Donau? 2. Kann die Bundesregierung Zeitungsberichte bestätigen oder dementieren, wonach Joseph Mengele während seines Besuches in Günzburg im Englischen Institut (Katholisches Mädcheninternat der Englischen Fräulein [...J) versteckt worden war?" Kleine Anfrage der Abgeordneten Frau Kelly und der Fraktion DIE GRÜNEN betr. Joseph Mengele und Besuch des Präsidenten Stroessner in der Bundesrepublik Deutschland (Bundestagsdrucksache 10/3033), 14. 3. 1985, in: Parlamentsspiegel (Internetdatenbank des Bundestages und der 16 Länderparlamente beim Landtag von Nordrhein-Westfalen), URL: http://www.parlamentsspiegel.de, Erstelldatum unbekannt, eingesehen am 28. 1. 2002.

Dazu kamen noch die Punkte 3 und 7, in denen Kelly sich erkundigte, was die Bundesregierung unternehme, um Mengele zu finden und ob sie das Mengele-Tribunal finanziell, politisch oder moralisch unterstützt habe.

133 In den Punkten 4-6 erkundigte sich Kelly, ob die Bundesregierung plane, mit Stroessner ausführlich über Mengele und Menschenrechtsverletzungen in Paraguay zu sprechen, ob es in diesem Zusammenhang Meinungsverschiedenheiten zwischen Kanzler Kohl und Bundesaußenminister Genscher gebe und wie die Einladung zustande gekommen sei. Vgl. ebenda.

134 Vgl. Petra Kelly jagt Mengele, in: Bild-Zeitung, 23. 3. 1985.

135 Lannion sorgt sich um Ruf der Partnerstadt, in: GZ, 2. 7. 1985.

136 Ebenda. 
Bundestagsabgeordnete konnte sich trotz des von ihr besonders hervorgehobenen persönlichen Charakters dieser Gespräche, die auch noch nicht allzu lange zurücklagen, nicht mehr erinnern, von wem sie die Informationen „unter [sic!] vorgehaltener Hand gehört" 137 hatte; Informationen immerhin, die sie nun für brisant genug hielt, die Bundesregierung damit zu befassen. Einzig „die verstorbene Mater Susanne“, die ja nun (zufällig?) als Zeugin nicht mehr zur Verfügung stand, konnte sie noch „bezeichnen“138. Erzählt hätten sie ihr jedenfalls, sie hätten „den Mengele abends? - nur in das Kloster hineinhuschen sehen" 139 .

$\mathrm{Ob}$ die Schwestern dies ihrer aus der katholischen Kirche ausgetretenen ${ }^{140} \mathrm{Ex}-$ Schülerin nun tatsächlich anvertrauten (was sie heftig dementierten ${ }^{141}$ ), muß letztlich offen bleiben. Eher dürfte die von Kelly kolportierte Version die Gerüchte widerspiegeln, die in Günzburg zu jener Zeit kursierten. Als weitere Quelle nannte Kelly in der Vernehmung durch die Staatsanwaltschaft eine „Stammtischrunde [...] nach einer Wahlveranstaltung so in der Zeit $80 / 81$ "142 und in einem späteren Leserbrief schrieb sie, sie sei während des Landtagswahlkampfs 1982 immer wieder auf diese Gerüchte angesprochen worden. ${ }^{143}$ In den politisch eher linksalternativen Kreisen, die als Klientel einer Wahlkampfveranstaltung der Grünen in den frühen 1980er Jahren in Frage kommen, wurde der Umgang der frühen Bundesrepublik mit der NS-Vergangenheit kritisch bewertet. Deshalb dürften diese Gerüchte dort schnell als Tatsachenberichte aufgefaßt worden sein, paßten sie doch zu gut ins weitgehend durchaus zutreffende - Bild von der Gleichgültigkeit der Gesellschaft der fünfziger Jahre gegenüber NS-Verbrechen und Tätern. Außerdem, so Kelly, habe sie bereits in verschiedenen Zeitungen davon gelesen. ${ }^{144}$ Das „offene Geheimnis" 145 , das sie in Günzburg zu Tage gefördert haben wollte, war also nichts, was als Gerücht nicht schon seit Jahrzehnten bekannt gewesen wäre ${ }^{146}$, erweitert um die wenig handfesten, dafür aber um so skandalträchtigeren Berichte der Nonnen - unabhängig davon, ob die Gespräche nun tatsächlich stattgefunden haben oder nicht.

137 Sta F/M, Az 4 Js 340/68, Fahnd.A., FO Deutschland III, Aussage Petra Kelly, 24. 5. 1985.

138 Ebenda.

139 Ebenda.

140 Vgl. AGG, PKA 79, Bl. 85, Schreiben Kelly an Höffner, 19. 6. 1985.

141 Vgl. Petra Kelly jagt Mengele, in: Bild-Zeitung, 23. 3. 1985 und Lannion sorgt sich um Ruf der Partnerstadt, in: GZ, 2. 7. 1985.

142 Sta F/M, Az 4 Js 340/68, Fahnd.A., FO Deutschland III, Aussage Petra Kelly, 24. 5. 1985. Auch die Teilnehmer an dem Stammtisch, die mit ihr gesprochen hatten, konnte sie „leider nicht namentlich bezeichnen“ - nur an einen „uralte[n] Chefredakteur der Günzburger Zeitung" könne sie sich erinnern.

143 Vgl. AGG, PKA 79, Bl. 91, Leserbrief Kelly an die GZ, 10. 7. 1985. Vgl. auch den gekürzten Abdruck: Wollte niemand verletzen, in: GZ, 16. 7. 1985.

144 Sie zitiert als Beispiele aus der Tat, 15. 1. 1982 und der Bild am Sonntag, 22. 6. 1980. Vgl. ebenda.

145 Ebenda.

146 Die Frage, woher das Detail, Mengele habe im Institut der Englischen Fräulein übernachtet, letztendlich stammt, muß offen bleiben. Als die Begräbnis-Legende die Medien 1964 erstmals beschäftigte, war davon noch keine Rede - und daß so ein pikantes Detail wie die Unterbringung des gesuchten Massenmörders in einem Nonnenkloster übersehen worden wäre, ist unwahrscheinlich. Erstmals tauchte sie, wie bereits erwähnt, 1967 in Wiesenthals Buch auf. Er berief sich dabei ausgerechnet auf den gleichen Gewährsmann wie im Falle von Mengeles angeblichem Aufenthalt auf der griechischen Insel Kythnos (vgl. Wiesenthal, Mörder, S. 204). 
Petra Kelly erinnerte sich an jenen angeblichen Aufenthalt Mengeles im Kloster zu einem Zeitpunkt, als vergangenheitspolitische Aspekte die innenpolitische Atmosphäre der Bundesrepublik Deutschland dominierten. Auf der tagespolitischen Agenda stand neben dem Besuch Stroessners auch der geplante Besuch Ronald Reagans und die Frage, ob der amerikanische Präsident eine KonzentrationslagerGedenkstätte (was dem Opferselbstbild vieler Deutscher widersprach), den Soldatenfriedhof Bitburg (auf dem auch SS-Angehörige bestattet waren) oder beides besuchen sollte. So ist es wenig erstaunlich, daß sich Petra Kelly gerade zu diesem Zeitpunkt jenes „offenen Geheimnisses“ erinnerte, das sie zunächst nicht für sonderlich wichtig gehalten haben konnte - andernfalls wären die häufigen Gedächtnislücken, nicht nur angesichts „sehr persönlich“ geführter Gespräche mit den Nonnen, doch sehr erstaunlich. Nun sah sie darin ein geeignetes Instrument, um die Bundesregierung im politischen Kampf anzugreifen, und man muß ihr nicht persönliche Rachegefühle gegenüber den Nonnen unterstellen, um diesen Sinneswandel zu erklären, wie dies eine ehemalige Mitschülerin getan hat. ${ }^{147}$ Eher zutreffend ist ihr Vorwurf der "Showmache“ 148 , die allerdings durchaus zum täglichen Brot des Politikers gezählt werden darf: Im weltweiten Medienrummel um den KZ-Arzt erinnerte sich die erfahrene Politikerin Kelly just dann an die Mengele-Episode, als sich die entsprechenden Schlagzeilen politisch ausschlachten ließen. Die grüne Abgeordnete hat die Günzburger Gerüchte instrumentalisiert und sie mit dem von ihrer Partei politisch bekämpften Stroessner-Besuch verknüpft, um von dem großen Medieninteresse am Fall Mengele und anderen vergangenheitspolitischen Fragen zu profitieren.

Noch deutlicher wurde der Versuch, die Begräbnislegende politischen Zwecken dienstbar zu machen, im Juni 1985. ${ }^{149}$ Auf dem Höhepunkt des Medienrummels um die Identifizierung der in Embu aufgefundenen Leiche wandte sich Kelly in einem offenen Brief an den Vorsitzenden der deutschen Bischofskonferenz, Erzbischof Joseph Kardinal Höffner. Offenbar wollte sie die günstige Gelegenheit nicht versäumen, der „katholischen Amtskirche"150 ihr Verhalten während der NS-Zeit („Nur in allgemeinen Wendungen von einer Mitschuld der Kirche zu sprechen, das reicht nicht aus, Kardinal Höffner"151) und den moralisch zweifelhaften Umgang mit NS-Verbrechern in der Nachkriegszeit vorzuwerfen. „Sollte [...] die Hierarchie der Kirche und des Klosters [...] über die Anwesenheit Josef Mengeles [...] 1959 in-

147 „Petra Kelly hatte damals einen schweren Stand, da ihre damaligen häuslichen Verhältnisse nicht ganz den Vorstellungen einer christlichen Familie entsprachen “. Leserbrief: Entsetzen über Kelly, in: GZ, 9. 7.1985.

148 Ebenda.

149 Zwischenzeitlich hatte die Bundesregierung Kelly in der Antwort auf ihre Anfrage beschieden, daß hinsichtlich des Besuchs Mengeles 1959 und seines angeblichen Versteckes keine Erkenntnisse vorlägen und wies außerdem ausdrücklich darauf hin, sie lege „Wert auf die Feststellung, daß sie mit der Beantwortung dieser Anfrage nicht dazu beitragen will, daß bestimmte Personen oder eine bestimmte Firma in ungerechtfertigten Mißkredit gebracht werden“. Antwort auf die Kleine Anfrage der Abgeordneten Frau Kelly und der Fraktion DIE GRÜNEN betr. Joseph Mengele und Besuch des Präsidenten Stroessner in der Bundesrepublik Deutschland (Bundestagsdrucksache 10/3130), 1. 4. 1985, in: Parlamentsspiegel (Internetdatenbank des Bundestages und der 16 Länderparlamente beim Landtag von Nordrhein-Westfalen), URL: http://www.parlamentsspiegel.de, Erstelldatum unbekannt, eingesehen am 28.1.2002.

150 Dieses und die folgenden Zitate: AGG, PKA 79, Bl. 85, Schreiben Kelly an Höffner, 19. 6. 1985.

151 Ebenda. 
formiert gewesen sein [...], so machen sich Kloster und Kirche [...] mitschuldig "152, schrieb Kelly. In der Tat war die Haltung der katholischen Kirche gegenüber den Kriegsverbrechern, die oft genug auf Fluchthilfe hinausgelaufen war, vielfach kritikwürdig. Doch Petra Kelly erhob den moralischen Zeigefinger in diesem Fall, obwohl sie noch einen Monat zuvor, am 24. Mai 1985, bei der Vernehmung durch die Staatsanwaltschaft sicher gewesen war, die Schwestern hätten gar nichts von der heimlichen Unterbringung Mengeles im Kloster gewußt. Dies hinderte sie nicht daran, die Amtskirche und das Kloster nun zu verdächtigen, „Mitwisser“ gewesen zu sein. Nach entsetzten Reaktionen aus Günzburg ${ }^{153}$ sah sie sich dann allerdings bald genötigt, zurückzurudern und zu dementieren. Es sei der Eindruck erweckt worden, so klagte sie in einem Leserbrief an die Günzburger Zeitung, sie verdächtige die Nonnen, Mengele „wissentlich Unterschlupf gewährt" zu haben: „Dies babe ich nie behauptet." 154 Sie tat so, als ob ihr der Vorwurf lediglich untergeschoben worden sei, doch sie selbst hatte in ihrem offenen Brief an Kardinal Höffner eben dies zwar nicht explizit behauptet, aber doch den Verdacht in die entsprechende Richtung gelenkt. Wozu sonst hätte dieses Schreiben dienen sollen? Ihre angeblich gegenteilige Überzeugung oder ihr besseres Wissen hatten die Politikerin offenbar nicht daran hindern können, der Versuchung nachzugeben und ihre Version des Günzburg-Mythos zu nutzen, um die katholische Amtskirche an den Pranger zu stellen.

Das Beispiel Petra Kellys zeigt, wie sehr sich im Windschatten gesteigerter öffentlicher Aufmerksamkeit Vergangenheit im tagespolitischen Kontext instrumentalisieren läßt. Sein Entkommen hatte den dämonisierten Mengele zu einem Mahnmal für die vielen NS-Verbrecher werden lassen, die sich durch Flucht der Gerechtigkeit entzogen hatten oder durch gesellschaftliches Desinteresse und juristische Versäumnisse keine Verfolgung fürchten mußten, und so ließ sich mit dem Symbol Mengele Politik machen gegen all diejenigen, die man damit irgendwie in Verbindung bringen zu können glaubte. Auch wenn die grüne Bundestagsabgeordnete den Höhepunkt dieser nicht uneigennützigen Moralpolitik des erhobenen Zeigefingers markierte, so war sie doch nicht die einzige, die sich den Fall Mengele und den Günzburg-Mythos nutzbar zu machen verstand, um für die eigenen politischen Ansichten und gegen den politischen Gegner größtmögliche Publizität zu erreichen. ${ }^{155}$

\section{Defensiostrategien}

Oberbürgermeister Köppler - Erfolgsrezept „Offensive Verteidigung“

Die Stadt war auch in den Jahren zuvor immer wieder mit dem Fall Mengele konfrontiert worden, doch noch nie war das Medieninteresse so gewaltig gewesen wie in den Wochen nach dem Mengele-Tribunal Anfang Februar 1985 in Jerusalem. Die GZ bediente sich einer geradezu kriegerischen Metaphorik: „Scharenweise sind Reporter aus dem In- und Ausland eingefallen. "156 Internationale Fernsehsender

152 Ebenda.

153 Vgl. Lannion sorgt sich um Ruf der Partnerstadt, in: GZ, 2. 7. 1985 und Leserbrief: Entsetzen über Kelly, in: GZ,9.7.1985.

154 Vgl. AGG, PKA 79, Bl. 91, Leserbrief Kelly an die GZ, 10. 7. 1985, Hervorhebung im Original. Vgl. auch den gekürzten Abdruck: Wollte niemand verletzen, in: GZ, 16. 7. 1985.

155 Vgl. auch S. $173 \mathrm{f}$.

156 Günzburg: Eine Stadt kämpft um ihren Ruf, in: GZ, 2./3.3. 1985. 
gaben sich im Rathaus beinahe schon die Klinke in die Hand, und Köppler fürchtete die weltweite Verbreitung der Legende, „daß Günzburg ein Hort unbelehrbarer Nazis ist, die hinter Mengele stehen "157. Das Beispiel des Daily Express - das sicherlich das extremste ist - bestätigte diese Befürchtung und zeigt gleichzeitig, wie wenig sich die Grundmuster des Günzburg-Mythos seit den 1960er Jahren verändert hatten. Viele der Journalisten kamen - wie in den 1960er Jahren - mit einer bereits weitgehend gefestigten Vorstellung in die Stadt, mit „vorgefaßten Meinungen und einem schrecklichen Bild im Kopf" 158 , wie Köppler es gegenüber der GZ ausdrückte. Auch das Verhalten vieler Günzburger stellte sich nach außen ähnlich dar wie zwei Jahrzehnte zuvor: Wieder wollten viele nicht mit den Reportern sprechen, weil „die Bürger es mittlerweile satt haben, als Komplizen eines Kriegsverbrechers geschildert zu werden"159. Viele Günzburger reagierten zunehmend gereizter auf den Versuch, sie „zur Rechenschaft“ zu ziehen „für einen Mann, der nur die ersten 19 Jahre seines Lebens hier verbracht hat" 160 . Ein Günzburger Stadtrat formulierte es so: „Zwischen Auschwitz und Günzburg [... g gibt es doch keine Verbindung." 161

Auch Köppler war nicht „willens hinzunehmen, daß der Name Günzburgs in Verbindung mit Mengele gebracht werde" 162 , weil er eine besondere Schuld seiner Stadt an dessen Verbrechen nicht erkennen konnte. Die Ereignisse der vorangegangenen Jahre hatten indes mehr als deutlich gezeigt, daß Günzburg die Tatsache nicht einfach würde ignorieren können, daß Josef Mengele dort geboren war. Deshalb entschied er sich, den Ruf seiner Stadt „offensiv zu verteidigen " 163 .

Grundlage von Köpplers Konzept der „offensiven Verteidigung“ war zunächst die Akzeptanz einer Sonderstellung Günzburgs im Fall Mengele als gegebenes Faktum - ohne sie deshalb als berechtigt anzuerkennen. Damit hatte er seine Haltung seit 1982 erkennbar geändert, als er den Mengele-Informationsstand Hanne Hiobs („Das geht Günzburg am meisten an“) noch mit den Worten kritisiert hatte: „Wenn's auch woanders gezeigt wird, ist das völlig legitim." 164 Ohne diese stillschweigende Akzeptanz hätte Köppler jedes Gespräch mit Journalisten als von vornherein gegenstandslos ablehnen müssen - wie es viele Günzburger ja tatsächlich taten - und hätte sich damit jeder Möglichkeit beraubt, das eigene Bild der Stadt zu kommunizieren und korrigierend auf die Berichterstattung einzuwirken. Eine solche Haltung wäre für das Ziel, das Bild der Stadt zu berichtigen, in jedem Falle nutzlos, eher sogar kontraproduktiv gewesen, da sie negative Erwartungen erfüllt und Vorurteile bestätigt hätte. Während des Besuchs Friedmans Anfang 1985 hatte Köppler bereits entsprechend gehandelt, die beiden Demonstranten im Rathaus empfangen und so die Gelegenheit gehabt, seinen eigenen Standpunkt darzulegen.

In der Phase des enormen internationalen Presseandrangs im Februar/März 1985 folgte er der neuen Strategie und stellte sich dem Gespräch mit den Medienvertre-

157 Ebenda.

158 Ebenda.

159 Ebenda.

160 Gestörte Idylle (2), in: DIE ZEIT, 26. 4. 1985.

161 Ebenda.

162 Köppler kämpft um den guten Ruf Günzburgs, in: GZ, 23./24. 2. 1985.

163 Günzburg: Eine Stadt kämpft um ihren Ruf, in: GZ, 2./3. 3. 1985.

164 Das geht Günzburg am meisten an, in: GZ, 24. 11. 1982. 
tern. Damit eröffnete sich ihm die Chance, in Interviews die alten Klischees des Günzburg-Mythos zu widerlegen oder zumindest eine allzu einseitige Berichterstattung zu verhindern und die Distanzierung der Stadt von den Taten des KZ-Arztes zu dokumentieren. Dabei legte er - wie in ähnlichen Situationen in den Jahren zuvor - viel Wert darauf zu betonen, daß es in Günzburg selbstverständlich Nationalsozialisten gegeben habe, die Stadt aber keine NS-Hochburg gewesen sei. Ebenfalls sei nicht von der Hand zu weisen, daß es in Günzburg „wie andernorts auch einen Bodensatz ewiggestriger Nazis“ 165 gebe und „ein paar, die aus der Geschichte nichts gelernt haben"166. Doch "Günzburg ist nicht schlimmer und besser als andere Städte auch“167, und Josef Mengele, „dieser verblendete Mann ohne Gespür für Gut und Böse und ohne moralische Skrupel hätte überall geboren werden können"168.

Köppler hatte mit seinem Konzept der offensiven Verteidigung zumindest teilweise Erfolg. Das Interview mit der amerikanischen ABC sei „fair"169 gewesen, verriet der Bürgermeister der GZ, und die anschließende Berichterstattung in den USA sei entsprechend gewesen. ${ }^{170}$ Auch wenn die Gespräche mit anderen Medienvertretern „wesentlich aggressiver" 171 verliefen und insbesondere Boulevardblätter wie der Daily Express sich nur für verkaufsfördernden Sensationsjournalismus interessierten und in Amerika viel von „mengele-town" 172 die Rede war, waren bald auch deutlich differenziertere Töne zu vernehmen. ${ }^{173}$ DIE ZEIT vermerkte lobend, der Oberbürgermeister weiche „keiner Frage aus, will die historische Wahrheit freigelegt wissen" 174 . Doch nicht nur sein Verhalten, sondern auch und gerade die Person Köpplers und seine persönliche Biographie erwiesen sich als Glücksfall für die Stadt. Im Gespräch mit den Journalisten konnte Köppler als gebürtiger Berliner und Sozialdemokrat auf einen gewissen Glaubwürdigkeitsbonus zählen. Gegen den Vorwurf, er betreibe das „Reinwaschen der Nazis und ihrer Handlanger vom Schlage Josef Mengeles" ${ }^{175}$, schützte ihn, dessen Vater gegen Kriegsende wegen Defaitismus und Wehrkraftzersetzung standrechtlich erschossen worden war, nicht zuletzt das persönliche Schicksal. Nun erwies sich als vorteilhaft, daß die Stadt schon in den Jahren zuvor immer wieder mit dem Fall Mengele konfrontiert worden war; insbesondere auf die Rede vor dem Stadtrat konnte Köppler immer wieder verweisen und so belegen, daß die Stadt sich durchaus mit ihrer Vergangenheit auseinandergesetzt habe.

165 Günzburg: Eine Stadt kämpft um ihren Ruf, in: GZ, 2./3.3. 1985.

166 Eine Stadt wehrt sich gegen einen monströsen Schatten, in: Die Welt, 7. 3. 1985. Als Beispiel führt Die Welt ,jenen Heimatdichter an, der Mengele für einen Märtyrer hält. Aber was der Oberbürgermeister über diesen Mitbürger sagt, bittet er, nicht zu schreiben, um einem Beleidigungsprozeß zu entgehen."

167 Ebenda.

168 Günzburg: Eine Stadt kämpft um ihren Ruf, in: GZ, 2./3.3. 1985.

169 Köppler kämpft um den guten Ruf Günzburgs, in: GZ, 23./24. 2. 1985.

170 Vgl. Günzburg: Eine Stadt kämpft um ihren Ruf, in: GZ, 2./3.3. 1985.

171 Köppler kämpft um den guten Ruf Günzburgs, in: GZ, 23./24. 2. 1985.

172 Vgl. „Falsche Fährten haben nur Nazi-Jäger gelegt“, in: GZ, 13. 6. 1985.

173 Vgl. z.B. Eine Stadt wehrt sich gegen einen monströsen Schatten, in: Die Welt, 7. 3. 1985 und Gestörte Idylle (2), in: Die Zeit, 26. 4. 1985.

174 Gestörte Idylle (2), in: Die Zeit, 26. 4. 1985.

175 Günzburg: Eine Stadt kämpft um ihren Ruf, in: GZ, 2./3.3. 1985. 
Familie Mengele - Schweigen, Täuschen, Tarnen

Während Köppler beinahe täglich von Journalisten aus aller Welt mit dem Fall Mengele konfrontiert wurde und auf ein differenzierteres Bild seiner Stadt hinarbeitete, wollten Karl-Heinz und Dieter Mengele vor allem „nicht belästigt “ ${ }^{176}$ werden. Sie setzten weiterhin auf konsequentes Schweigen und hielten jede Stellungnahme für „völlig sinnlos“177. Der Oberbürgermeister hatte für diese Haltung wenig Verständnis und ließ deutliche Kritik am Verhalten der Familie Mengele erkennen. Zwar war er immer noch fest davon überzeugt, daß „keine Verbindungen Mengeles nach Günzburg bestünden" 178 und verteidigte die Familie auch weiterhin gegen eine vermeintliche „Sippenhaftung“, doch gerade weil die beiden Firmenerben „zu jung sind, um mit Nationalsozialismus und Judenverfolgung in Verbindung gebracht zu werden "179, konnte der Oberbürgermeister ihre Blockadehaltung nicht nachvollziehen. Während er um den Ruf Günzburgs kämpfte, trieb die Familie, die doch angeblich nichts zu verbergen hatte, „ein ihm unverständliches Versteckspiel“ 180 .

Lediglich einmal sahen sich Karl-Heinz und Dieter Mengele gezwungen, ihr Schweigen zu brechen und sich zu ihrem Verhältnis zu ihrem Onkel zu äußern. Ende Februar war der Bayerische Ministerpräsident Franz Josef Strauß während eines Israel-Besuchs mit dem Fall Mengele konfrontiert worden. Ein israelischer Reporter hatte behauptet, daß "Gewinne und Dividenden der Firma [...] auf ein Schweizer Konto von Josef Mengele überwiesen "181 würden. Strauß wollte in Tel Aviv erstmals von der Verbindung des KZ-Arztes mit der Günzburger Firma gehört haben und versprach dem Fragesteller eine Untersuchung.

„Die ,Untersuchung' war kurz, bündig und ergebnislos" 182 , spottete wenige Tage später die Süddeutsche Zeitung. Ein Sprecher der Staatskanzlei hatte mitgeteilt, es gebe „keine Anhaltspunkte dafür, daß Josef Mengele aus der Firma in Günzburg Überweisungen auf ein Schweizer Nummernkonto erhalte"; es sei allerdings schlicht unmöglich, zu überprüfen, ob er „Zuwendungen aus Günzburg erhalte“, denn „wegen des Steuergeheimnisses habe Strauß ,überhaupt keine Handhabe“". „Da wird nichts herauskommen“, so das Fazit der Staatskanzlei. „Die Untersuchung hat also ergeben, daß eine Untersuchung sinnlos [...] ist", kommentierte die Süddeutsche spöttisch. Die Opposition im Landtag wollte sich damit nicht zufrieden geben: Der bayerische SPD-Landtagsabgeordnete Klaus Warnecke erkundigte sich in einer Anfrage, „ob bayerische Behörden jemals den Versuch unternommen haben, das in Bayern vorhandene Vermögen von Mengele für die Entschädigung von KZ-Opfern [...] sicherzustellen“ 183 . Selbst wenn Mengele heute nicht mehr der Eigentümer sei, so stand für Warnecke doch fest, „daß Mengele einst Besitzer oder Mitbesitzer der Firma war" 184 .

176 Köppler kämpft um den guten Ruf Günzburgs, in: GZ, 23./24. 2. 1985.

177 Ebenda.

178 Ebenda.

179 Eine Stadt wehrt sich gegen einen monströsen Schatten, in: Die Welt, 7. 3. 1985.

180 Ebenda.

181 Ebenda.

182 Dieses und die folgenden Zitate: „Da wird nichts herauskommen“, in: SZ, 6. 3. 1985.

183 Mengele Fabrikbesitzer in Bayern?, in: Sozialdemokratischer Pressedienst, 5. 3. 1985.

184 Ebenda. 
Nun war die Vermutung nicht neu, Josef Mengele sei immer noch an dem Günzburger Unternehmen beteiligt und finanziere seine spektakuläre Flucht aus den Gewinnen der Firma. Doch durch die enorm gestiegene Aufmerksamkeit, die der Fall Mengele mittlerweile weltweit auf sich zog, hatten sich die Rahmenbedingungen schlagartig geändert. Nach dem medienwirksamen Versprechen von Strauß in Israel und der folgenden, nur halbherzigen Entlastung, die eher einem wackeligen "aus Mangel an Beweisen" als einem soliden "nicht schuldig“ glich, drohte das weltweite Image der Firma weiteren, ernsthaften Schaden zu nehmen, zumal die parlamentarische Opposition nicht nur im bayerischen Landtag, sondern auch im Bundestag das Thema aufgegriffen hatte. ${ }^{185}$

In dieser Situation sahen sich Karl-Heinz und Dieter Mengele zu einer Stellungnahme genötigt. Diese erfolgte nun eindeutig aus der Defensive; die Chance, ähnlich wie Köppler durch einen offeneren Umgang mit dem Thema Glaubwürdigkeit zu gewinnen, war verpaßt. Er habe, so Karl-Heinz Mengele, „mit Josef Mengele niemals Kontakt gehabt [und] ihn nie bewußt zu Gesicht bekommen“ - an den Skiurlaub in der Schweiz und die Jahre in Argentinien wollte sich der Stiefsohn öffentlich nicht erinnern. Die Vorwürfe, die Firma oder die Familie unterstütze Josef Mengele, seien „erstunken und erlogen“: „Wir zahlen nichts, und wir haben nichts bezahlt, auch nicht auf ein Schweizer Nummernkonto."186

Die Aussage, Josef Mengele sei „nie an der Firma beteiligt" 187 gewesen, entsprach tatsächlich der Wahrheit und war auch leicht zu belegen. ${ }^{188}$ Ansonsten beriefen sich Karl-Heinz und Dieter Mengele immer wieder auf ihr Alter: Die ganze Angelegenheit sei schließlich eine Sache, "mit der wir schon wegen unseres Alters nichts zu tun haben können" ${ }^{189}$. Diese Argumentation ging jedoch an der Natur der Verdächtigungen vorbei. Schließlich beschuldigte die Cousins ja niemand der Mittäterschaft

185 Neben der parlamentarischen Anfrage des bayerischen Landtagsabgeordneten Warnecke hatten auch die Bundestagsabgeordneten Schöfberger und Schmidt (beide SPD) am 8. März drei Anfragen im Zusammenhang mit dem Fall Mengele an die Bundesregierung gerichtet. Eine davon zielte auf die finanziellen Verbindungen Josef Mengeles zur Günzburger Firma. Vgl. Anfragen Nr. 85-87 für die Fragestunden der Sitzungen des Deutschen Bundestages am 13. 3. 1985 und 14. 3. 1985 (Bundestagsdrucksache 10/2987, S. 16), 8. 3. 1985, in: Parlamentsspiegel (Internetdatenbank des Bundestages und der 16 Länderparlamente beim Landtag von Nordrhein-Westfalen), URL: http://www.parlamentsspiegel.de, Erstelldatum unbekannt, eingesehen am 28.1.2002.

186 Mengele-Verwandter stellt klar: „Wir zahlen nichts“, in: GZ, 6. 3. 1985. Das plakative Zitat wurde vielfach verwendet, so z. B. außerdem in: Onkel Josef war nie an der Firma beteiligt, in: Südwest-Presse, 6. 3. 1985; „Da wird nichts herauskommen“, in: SZ, 6. 3.1985 und Gestörte Idylle (2), in: Die Zeit, 26. 4. 1985.

187 Mengele-Verwandter stellt klar: „Wir zahlen nichts“, in: GZ, 6. 3. 1985.

188 Josef Mengele hatte 1949, noch vor seiner Flucht nach Südamerika, auf alle Erbansprüche verzichtet. Vgl. S. 49. Dies war bereits in den 1960er-Jahren nach „einem Artikel in einer Hamburger Illustrierten" gerichtlich festgestellt worden. Vgl. Onkel Josef war nie an der Firma beteiligt, in: Südwest-Presse, 6. 3. 1985. Bei dem gerichtsnotorischen Artikel dürfte es sich um den Beitrag „Kein Dankeschön für Mord“ (in: Stern, 26. 11. 1961) gehandelt haben, in dem es hieß: „,Karl Mengele \& Söhne ‘... und Söhne. Das war ein Schock für uns - bedeutet es doch, daß [...] Dr. Josef Mengele heute gesetzlicher Mitinhaber des Landwirtschafts-Maschinenwerks in Günzburg ist." Vgl. außerdem Sta F/M, Az 4 Js 340/68, HandA. Bd. I, Bl. 130, Schreiben des Direktors des Amtsgerichtes Günzburg an die Sta Freiburg/ Breisgau betr. Erbangelegenheit Karl Mengele, 15. 1. 1960.

189 Mengele-Verwandter stcllt klar: „Wir zahlen nichts“, in: GZ, 6. 3. 1985. 
in Auschwitz, für die sie zweifelsohne zu jung waren oder wollte sie an ihres Onkels statt dafür zur Verantwortung ziehen. Vielmehr ging es um eine mögliche Unterstützung des KZ-Arztes durch seine Angehörigen, und dafür gab es natürlich keine Altersgrenze. Diesem Vorwurf hätte nur durch eine eindeutige Distanzierung von Josef Mengele und seinen Taten zumindest zum Teil begegnet werden können, doch die Mengeles nahmen auch jetzt die Gelegenheit nicht wahr. Statt dessen wurden in der ersten Stellungnahme der Familie seit langem die Verbrechen Mengeles relativiert und in Zweifel gezogen: Zwar, so Karl-Heinz Mengele, habe sein Stiefvater „Schuld auf sich geladen [...] Auschwitz hat existiert, es sind ungeheuerliche Dinge passiert" 190 . Aber Beweise und offizielle Dokumente, die gegen Josef Mengele sprächen, seien dünn gesät, und es gebe Aussagen von ehemaligen Häftlingen, die ihn entlasteten: „Ich glaube deshalb nicht, daß alles so war, wie es jetzt [...] dargestellt wird." 191 Die Frage, ob der KZ-Arzt sich dann nicht endlich dem Gericht stellen sollte, wollte der Stiefsohn und Neffe „nicht beurteilen“192.

Damit hatte sich die Familie erneut mehr oder weniger deutlich vor den KZ-Arzt gestellt. Eine frühzeitige und unmißverständliche Verurteilung seiner Taten hätte möglicherweise weitere Imageschäden für die Firma im Ausland, die sowohl die Mengeles als auch die Belegschaft ${ }^{193}$ fürchteten, abgewendet oder begrenzt, ohne daß damit natürlich das moralische Dilemma aufgelöst worden wäre. Statt dessen hatte die Familie lange versucht, das Thema totzuschweigen - ein Versuch, der angesichts des nun herrschenden enormen Medieninteresses endgültig zum Scheitern verurteilt war. Dies hatten die Mengeles nach dem Israel-Besuch des bayerischen Ministerpräsidenten und seinen Folgen offenbar erkannt. Nun versuchten sie auf andere Weise, der Suche nach Josef Mengele etwas Wind aus den Segeln zu nehmen: Zum einen durch den fast schon naiven Versuch, als Fürsprecher des Onkels aufzutreten nach dem Motto, so schlimm sei alles doch gar nicht gewesen; zum anderen durch behutsame Andeutungen in Richtung Wahrheit: Die beiden Cousins teilten mit, daß sie davon ausgingen, daß Josef Mengele längst tot sei, denn ansonsten wäre er „doch schon aufgespürt worden" 194 . Für ein derartiges Experiment war es natürlich längst zu spät, die nichtssagend begründete Andeutung wurde, sofern sie denn überhaupt wahrgenommen wurde, nach dem langen Schweigen und den relativierenden Ausführungen zu Mengeles Schuld als Schutzbehauptung und durchsichtiges Ablenkungsmanöver interpretiert. Auch die Ermittlungsbehörden hielten die Aussage für kaum glaubwürdig und werteten sie als Versuch, die Fahnder zu irritieren. ${ }^{195}$ Dennoch gaben die Cousins nicht auf und starteten einen weiteren Versuch, diesmal in einem Interview mit John Martin von der ABC, das aber ebensowenig konkret, damit ebensowenig glaubwürdig und in der Konsequenz ebensowenig erfolgreich war. ${ }^{196}$ Alles deutete auf eine falsche Fährte hin, die den KZ-Arzt von den Nachstellungen seiner mittlerweile so zielstrebig wie noch nie vorgehenden Jäger entlasten sollte.

190 Ebenda.

191 Ebenda.

192 Ebenda.

193 Vgl. Köppler kämpft um den guten Ruf Günzburgs, in: GZ, 23./24. 2. 1985 und Gestörte Idylle (2), in: Die Zeit, 26. 4. 1985.

194 Mengele-Verwandter stellt klar: „Wir zahlen nichts“, in: GZ, 6. 3. 1985.

195 „Falsche Fährten haben nur Nazi-Jäger gelegt“, in: GZ, 13. 6. 1985.

196 Vgl. den Abdruck des betreffenden Teils des Interviews in: Posner/Ware, Mengele, S. 373. 


\section{Nach dem Fund von Embu: Reaktionen auf die Wabrheit}

\section{Die Mengeles in Günzburg - Die Blockadehaltung geht weiter}

Realiter mußte natürlich damit gerechnet werden, daß der mittlerweile 75-jährige Josef Mengele nicht mehr am Leben war. Die meisten, die mit dem Fall zu tun hatten, waren aber davon überzeugt, daß der Tod Mengeles auf jeden Fall bekannt geworden wäre: Weder Diktator Stroessner in Paraguay, unter dessen Schutz man Mengele noch immer vermutete, noch die Familie, von der man annahm, daß sie über Mengeles Aufenthaltsort und Schicksal bestens informiert war, konnten ein Interesse daran haben, sein Ableben zu verheimlichen und damit die Jagd und das internationale Medieninteresse künstlich zu verlängern.

Dementsprechend groß war das Erstaunen, als die erst wenige Monate zuvor intensivierten Ermittlungen zum Ziel führten und am 6. Juni 1985 sensationell gemeldet wurde, die Leiche Josef Mengeles sei auf dem Friedhof von Embu in Brasilien entdeckt worden. ${ }^{197}$ Die näheren Umstände, darunter auch die Mitteilung, daß der entscheidende Hinweis bei einer Hausdurchsuchung in Günzburg gefunden worden sei, teilte die Staatsanwaltschaft Frankfurt am nächsten Tag in einer Presseerklärung mit. ${ }^{198}$ Sowohl Rolf Mengele als auch die Familie in Günzburg hielten zunächst ihr Schweigen aufrecht. ${ }^{199}$ Erst am 11. Juni, vier Tage nach dem Fund, trat Rolf Mengele an die Öffentlichkeit und ließ eine kurze, kaum 16-zeilige Erklärung verlesen, in der es hieß, er sei vom Tod seines Vaters zweifelsfrei überzeugt. ${ }^{200} \mathrm{Zu}$ der Frage, „ob die Familie Mengele bis 1979 von Günzburg aus noch Kontakte mit Josef Mengele hatte“201, wollten sich die Sprecher Rolf Mengeles ${ }^{202}$, die die Erklärung in München verlasen, nicht äußern.

Am gleichen Tag erklärte sich auch die Familie in Günzburg. Man habe den Tod Josef Mengeles so lange verschwiegen, um „die Leute, die unserem Onkel 30 Jahre lang geholfen haben, $[z u]$ schützen: Wir wollten nicht, daß sie nachträglich für ihre Hilfsbereitschaft in die Pfanne gehauen werden"203. So recht verstehen wollten die beiden Cousins ohnehin nicht, warum ihnen dies nun zum Vorwurf gemacht

197 Vgl. S. 60 f.

198 Vgl. z.B.: Mengele: Briefe führten zum Grab nach Brasilien, in: Abendzeitung, 7. 6. 1985; Angeblicher Leichnam Josef Mengeles exhumieret, in: SZ, 8./9. 6. 1985; Endet die Jagd am Grab in Brasilien?, Spuren von und nach Günzburg; Spuren vom „Todesengel“, in: GZ, 8./9.6. 1985.

199 Vgl. die Äußerungen Simon Wiesenthals und Beate Klarsfelds z.B. in: Angeblicher Leichnam Josef Mengeles exhumiert, in: SZ, 8./9. 6. 1985; Im Haß vieler Menschen lebt dieser Mann weiter, in: Südwestpresse, 8.6. 1985; Spuren von und nach Günzburg, in: GZ, 8./9.1985.

200 Er teilte mit, er „habe keinen Zweifel daran, daß es sich bei dem am 5. Juni auf dem Friedhof in Embu/Brasilien exhumierten Leichnam um die sterblichen Überreste meines Vaters, Josef Mengele, handelt [...] Ich habe mit Rücksicht auf die Menschen, die mit meinem Vater in den letzten 30 Jahren in Beziehung standen, bisher geschwiegen. Allen Opfern und ihren Angehörigen gilt mein und unser tiefstes Mitgefühl“. Zit. nach: Rolf Mengele bestätigt den Tod seines Vaters, in: SZ, 12. 6. 1985. Vgl. auch: Gespannte Erwartungen im Fall Mengele, in: SZ, 11. 6. 1985; Familie bestätigt den Tod Josef Mengeles; Der Sohn will die Wahrheit jetzt ans Licht bringen, in: GZ, 12.6. 1985.

201 Der Sohn will jetzt die Wahrheit ans Licht bringen, in: GZ, 12. 6. 1985.

202 Die Schwägerin und ein Freund Rolf Mengeles.

203 „Falsche Fährten haben nur Nazi-Jäger gelegt“, in: GZ, 13. 6. 1985. 
wurde, schließlich hätten sie doch mehrfach „angedeutet, daß er tot ist. Aber wir können niemanden zwingen, uns zu glauben." $204 \mathrm{Daß}$ man konkretes Material schuldig geblieben sei, mußte Dieter Mengele einräumen, und daß diese vagen Andeutungen erst in den letzten Monaten unter dem immer größeren Druck des Medieninteresses abgegeben worden waren, verstärkte den Eindruck des Versuchs, sich herauszureden. Nicht ohne eine gewisse Schadenfreude fügte der Neffe des KZ-Arztes hinzu, der „ganze Käse von den angeblichen Millionen, mit denen Josef Mengele in Luxusvillen und beschützt von Leibwächtern lebte", sei nun wohl endgültig passé, und diese falschen Fährten hätte nicht die Familie, sondern "die sogenannten Nazi-Jäger“205 gelegt. Er sprach von einem „Märchenwald des Herrn Wiesenthal und der Frau Klarsfeld"206, doch auch das konnte kaum die Tatsache verschleiern, daß die Familie in Günzburg all die Jahre über gewußt hatte, wo Josef Mengele sich aufgehalten hatte. Daran konnte es nach der Hausdurchsuchung bei Hans Sedlmeier kaum noch vernünftige Zweifel geben.

Doch Karl-Heinz und Dieter Mengele hielten weiterhin an ihrer Verschleierungstaktik fest. Statt die jahrzehntelangen Verbindungen und Geldtransfers zwischen Günzburg und Südamerika endlich offenzulegen, wollten sie die Welt weiterhin davon überzeugen, daß es „Geld aus Günzburg [...] niemals für ihn gegeben“207 habe. Sogar, daß es überhaupt Kontakte gegeben habe, wurde weiterhin dementiert. ${ }^{208}$ Erneut war es Rolf Mengele, der offensichtlich nicht das beste Verhältnis zum Rest der Familie in Günzburg und deshalb auch keinen Grund zu weiterer Rücksichtnahme hatte ${ }^{209}$, der die Flucht nach vorne antrat: Schon wenige Tage nach den Verlautbarungen der Günzburger Cousins wurde bekannt, daß er die Geschichte der Flucht seines Vaters der Illustrierten „Bunte“ gratis angeboten hatte, wohl um in einem endgültigen Befreiungsschlag den Druck, der auf ihm und seiner Familie in Freiburg lastete, zu vermindern. ${ }^{210}$ Der Sohn bestätigte jedenfalls das Offensichtliche und widersprach seinen Cousins: Die Familie in Deutschland habe „immer gewußt, wo Josef Mengele war" und „habe dem Vater Geld geschickt, er müsse zwischen 200 und 500 Mark zum Leben gehabt haben"211.

Damit hatten die Mengeles auch in Günzburg jeden Rest an Glaubwürdigkeit verspielt. Der immer wieder unternommene Versuch, zu verschleiern und Nebelkerzen zu werfen, in der Hoffnung, die Angelegenheit werde früher oder später im Sande verlaufen, war auf ganzer Linie gescheitert. Dieter und Karl-Heinz-Mengele hatten geschwiegen, bis ein Ereignis, ein „Skandal“ sie zwang, Stellung zu beziehen,

204 Ebenda.

205 Ebenda.

206 Köppler verurteilt das lange Zögern, in: GZ, 13. 6. 1985.

207 „Falsche Fährten haben nur Nazi-Jäger gelegt“, in: GZ, 13. 6. 1985.

208 Vgl. Köppler verurteilt das lange Zögern, in: GZ, 13. 6. 1985.

209 Vgl. Um keinen Preis hätte er Mengele je verraten, in: GZ, 6./7. 7. 1985.

210 Rolf Mengele und seine Familie erhielten mittlerweile auch Morddrohungen. Vgl. Der Sohn will die Wahrheit jetzt ans Licht bringen, in: GZ, 12. 6. 1985.

Über die Motive Rolf Mengeles, mögliche Parallelen zu den gefälschten Hitler-Tagebüchern, auf die der Stern hereingefallen war und ethisch-moralische Bedenken wurde viel diskutiert. Vgl. z. B. Wem nützt der dicke Knüller, in: SZ, 20.6. 1985; Rolf Mengele: Die Familie schickte Geld, in: GZ, 19.6. 1985. Die Serie in der Bunten erstreckte sich über insgesamt fünf Ausgaben, in: Bunte, 20. 6., 27. 6., 4. 7., 11. 7. und 18. 7. 1985.

211 Rolf Mengelc: Die Familie schickte Geld, in: GZ, 19. 6. 1985. 
um dann so viel wie irgend möglich abzustreiten und weiterhin zu schweigen. Dies sollte sich auch jetzt nicht ändern: Zu Rolfs Angaben, die Familie habe Josef Mengele bis zum Schluß unterstützt, lehnte Karl-Heinz Mengele „jeden Kommentar $a b$ “212, hoffte, „daß endlich Ruhe einkehrt" und ließ sich nur zu dem Kommentar hinreißen: „Der Rolf muß wissen, was er tut.“213

\section{„Eine Belastung für die Stadt“ - Distanzierung von der Familie Mengele}

Nach der Exhumierung der Leiche, deren Identität zunächst ja noch zweifelhaft war, hatte in Günzburg ein Medienandrang eingesetzt, der die erste Welle im Februar und März des Jahres noch bei weitem übertraf214: „Journalisten aus Nordund Südamerika, aus Westeuropa und der ganzen Bundesrepublik [...] belagern regelmäßig das Werkstor der Landmaschinenfabrik, die Häuser von Karl-Heinz und Dieter Mengele sowie Hans Sedlmeier“215 und wieder einmal wurde „auf dem Marktplatz Volkes Stimme und Stimmung für die Nachrichtensendungen “216 eingeholt.

Die Prophezeiung des Frankfurter Staatsanwaltes Klein, der im März die Verstärkung der Fahndungsbemühungen „im nordschwäbischen Raum“217 mit dem Satz begründet hatte: „Wenn einer was weiß, dann sitzt er hier“, hatten sich erfüllt. Der "Schlüssel zu Josef Mengele" 218 hatte sich in dessen Heimatstadt gefunden. Der worst case, den Köppler immer befürchtet hatte, war eingetreten: „Nichts wäre schlimmer, als wenn weltweit behauptet werden könnte, in Günzburg versteckt man etwas." 219 Schnell stand zweifelsfrei fest, daß die „Familie Mengele, die in den vergangenen Jahren so häufig über die ihr zugefügte ,Sippenhaftung' geklagt

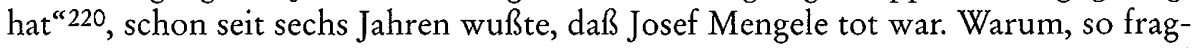
ten sich die Günzburger irritiert, hatte die Familie nicht schon vor Jahren den Tod des Gesuchten mitgeteilt und sich und der Stadt vieles erspart?221 Der SPD-Ortsvorsitzende Karl-Theodor Engelhardt sagte der GZ, er habe Karl-Heinz Mengele schon früher geraten, „sich möglichst rasch von den Verbrechen zu distanzieren“, und sein Kollege von der CSU, Günter Treutlein, meinte, das sei eine „persönliche Entscheidung“" gewesen, deren Tragweite der Familie, „die in der letzten Zeit genügend [babe] aushalten müssen“, bewußt gewesen sein müsse; für die Stadt sei das ganze sicherlich „nicht gewinnbringend“222 gewesen.

212 Ebenda.

213 Um keinen Preis hätte er Mengele verraten, in: GZ, 6./7.7. 1985.

214 Das generelle Interesse am Fall Mengele war auch weiterhin auf hohem Niveau geblieben, nicht zuletzt wachgehalten durch die in den USA laufenden Untersuchungen des OSI. Vgl. z.B. die Berichte zu Mengele aus den Monaten April und Mai: „Sechs Millionen, da kann ich nur lachen", in: Der Spiegel, 22. 4. 1985; Die neue Spur des Dr. Mengele, in: Stern, 25. 4. 1985; Hunting the „Angel of Death“, in: Newsweek, 20. 5. 1985.

215 Ebenda.

216 Ebenda.

217 „Wenn ciner was weiß, dann sitzt er hier“, in: GZ, 16./17. 3. 1985.

218 In Amerika sehr gefragt, in: GZ, 13.6. 1985.

219 Köppler kämpft um den guten Ruf Günzburgs, in: GZ, 23./24. 2. 1985.

220 „Falsche Fährten haben nur Nazi-Jäger gelegt", in: GZ, 13. 6. 1985.

221 Vgl. ebenda.

222 Ebenda. 
Oberbürgermeister Köppler wurde deutlicher und nannte das Verhalten der Mengeles eine „Belastung“223 für die Stadt. „Ich muß gestehen, ich bin bestürzt über das, was sich jetzt herausstellt. Daß doch ein halbes Dutzend Leute hier die ganze Zeit gewußt haben, was mit Josef Mengele war, die Sedlmeiers und die Mengeles, daß sie Kontakt gehabt haben, obwohl sie ihn immer geleugnet haben. Und ich habe immer Verständnis gehabt, wenn sich die Verwandten dagegen verwahrt haben, mit in den Strudel hineingezogen zu werden, ich bin immer davon ausgegangen, daß ihre Aussagen stimmen, daß sie nichts von Josef Mengele wissen."224 Nachdem er sich jahrelang vor die Familie gestellt und die Neffen, die doch nichts für ihren Onkel könnten, verteidigt hatte, habe er nach Bekanntwerden der Neuigkeiten nicht mehr mit der Familie gesprochen, so Köppler: Seine Aufgabe sei es, die Stadt zu verteidigen, ,alles andere ist jetzt Sache der Justiz“. Nicht nur er selbst, sondern viele Menschen in Günzburg seien „enttäuscht und verärgert. Die Familie hätte der Stadt sehr viel ersparen können, wenn sie schon 1979 diese Nachricht bekanntgegeben hätte“. Besonders bestürzt hatte Köppler, daß die Mengeles noch vor wenigen Monaten vorgegeben hätten, nichts über das Schicksal ihres Verwandten zu wissen: „Das muß man sich mal vorstellen.“

„Erste Anzeichen“ dafür, daß „das Verhalten der Familie Mengele, die jahrelange Deckung auch eines Günzburger ,Verbindungsmannes', die dubiose Informationspolitik der Betroffenen“ sich „noch negativ für Günzburg auswirken werde“225, gebe es bereits, unkte die Günzburger Zeitung am 15. Juni. „Der Flurschaden könnte einmal groß werden“, so die Befürchtung, „für die Menschen dieser Stadt und der Umgebung"; schließlich seien schon die ersten Auftragsstornierungen eingegangen, und „sollte dieses Beispiel Schule machen, [könnten] die Folgen [... J vernichtend sein“226.

Niemand, so Gernot Römer in einem Kommentar, habe „das Recht, es der Familie anzulasten, daß sie den Fahndungsbehörden vor 1979 nie einen Hinweis [...] gab“227. Natürlich sei es ein schweres Erbe, „Sohn oder enger Verwandter des furchtbaren Josef Mengele zu sein“. Auf einem anderen Blatt stehe dagegen, daß die Familie in Günzburg - nicht Rolf Mengele - den KZ-Arzt finanziell unterstützt habe. Sie „wird sich mit dem moralischen Vorwurf auseinandersetzen müssen, aktiv einem Mann geholfen zu haben, der unter dem Verdacht vieltausendfachen Mordes und unmenschlicher Experimente steht". Es bleibe "das Geheimnis der MengeleAngehörigen, warum sie damals den Tod verschwiegen“, obwohl doch „damals bereits der jetzt so beklagte Druck der Öffentlichkeit von der Familie" hätte genommen werden können.228

Jetzt sei „das Kind [...] praktisch schon in den Brunnen gefallen“, so ein anderer Kommentator, „die Chance einer gewissen Rehabilitierung böte nur noch die Of-

223 Köppler verurteilt das lange Zögern, in: GZ, 13. 6. 1985.

224 Dieses und die folgenden Zitate: Eine Kleinstadt ist verärgert (Ulrike Südmeyer, AP), Zeitungsartikel ohne Quellenangabe und Datum [7/1985], in: StAGz 130/1 (12), Spezialakt Josef Mengele, Bd. IV.

225 Wochennachlese: Hoch gepokert, in: GZ, 15./16. 6. 1985.

226 Ebenda.

227 Dieses und die folgenden Zitate: Fall Mengele schwelt weiter, in: GZ, 24. 6. 1985.

228 Gernot Römer irrt allerdings, wenn er schreibt, die Justiz hätte Mengeles Helfern damals nichts anhaben können. Zumindest Hans Sedlmeier wäre wohl wegen Strafvereitelung, die nach fünf Jahren verjährt, bis 1984 durchaus zu belangen gewesen. Die Familie genoß Zeugnisverweigerungsrecht. 
fenlegung bislang versteckten Materials und verborgenen Wissens"229. Dieser am 14. Juni geäußerte Wunsch sollte nicht in Erfüllung gehen - zu diesem Zeitpunkt leugneten die Mengeles immer noch hartnäckig, daß die Familie Josef Mengele unterstützt habe. Zwar sorgten sich viele Günzburger um den Ruf der Landmaschinenfabrik und um die Arbeitsplätze, wenn aber ausgerechnet Karl-Heinz Mengele über die „Gefahr der Firmenrufschädigung" klagte und bereits am 6. Juli meinte, die Günzburger Familienangehörigen hätten „mit dem Fall nichts mehr zu tun“, so zeugte dies von einer mangelnden Unterscheidungsfähigkeit zwischen Ursache und Wirkung sowie von einer bemerkenswerten Realitätsverweigerung. 230 Die Günzburger waren sich durchaus bewußt, daß die Mengeles bereit gewesen waren, „im Interesse einiger weniger, die ,Josef Mengele 30 Jahre geholfen haben' $[. .$.$] einen$ derart hohen Preis zu riskieren, wie er nun vermutlich von Günzburg und seinen Bürgern eingetrieben wird."231 Oberbürgermeister Köppler warf der Familie vor, sie habe „sich selbst und ihrem Unternehmen den denkbar schlechtesten Dienst erwiesen“, denn „wer die Justiz sechs Jahre lang in die Irre führe und nach einem Phantom fahnden lasse“, dürfe sich über „negative Folgen im Geschäftsleben“232 nicht wundern. Dabei übersah er allerdings, daß die Mengeles schon längst zu Gefangenen ihres eigenen Schweigens geworden waren: Zum einen sahen sie durch eine Offenlegung der Tatsachen die Reputation von Familie und Firma gefährdet; zum anderen ging es darum, den loyalen Hans Sedlmeier zu schützen. In seinem Fall stand nicht nur das persönliche Ansehen auf dem Spiel, denn - anders als den Familienmitgliedern - drohten ihm auch juristische Konsequenzen: Der Vorwurf der Strafvereitelung, dem er sich ausgesetzt hatte, war erst im Februar 1984, fünf Jahre nach dem Tod Mengeles, verjährt.

Wie groß der materielle Schaden war, den die Affäre der Firma Mengele kurzund längerfristig zugefügt hat, ist nicht zu überblicken. Der CSU-Landtagsabgeordnete Karl Kling sah den Betrieb in einer „bedrängten Situation“, und mit ihm eine Vielzahl von Zulieferfirmen, die nun „in den Strudel des verhängnisvollen Schweigens der Familie"233 zu geraten drohten. Karl-Heinz Mengele sah dies anders. Überlegungen, die Firma solle ihren Namen ändern, wies er zurück, mußte aber gleichzeitig eingestehen, daß für 1985 mit deutlichen Umsatzeinbußen zu rechnen sei. Diese führte der Firmenchef aber nicht auf den Skandal zurück, sondern auf die prekäre Lage der bäuerlichen Kundschaft, die nicht mehr investiere. Sechs Jahre später, als Karl-Heinz und Dieter Mengele schließlich aufgeben und die Firma verkaufen mußten, hatte sich die Mitarbeiterzahl von 1300 im Jahr 1985 auf nur noch 650 halbiert. ${ }^{234}$

229 Wochennachlese: Hoch gepokert, in: GZ, 15./16. 6. 1985.

230 Um keinen Preis hätte er Mengele verraten, in: GZ, 6./7. 7. 1985.

231 Wochennachlese: Hoch gepokert, in: GZ, 15./16. 6. 1985.

232 Schwere Hypothek, in: Wirtschaftswoche, 20. 9. 1985.

233 Ebenda.

234 Vgl. S. 107. 